\title{
A Review of Smart Homes in Healthcare
}

\author{
Mohsen Amiribesheli • Asma Benmansour • Abdelhamid Bouchachia
}

Received: date / Accepted: date

\begin{abstract}
The technology of Smart Homes (SH), as an instance of ambient assisted living technologies, is designed to assist the homes' residents accomplishing their daily-living activities and thus having a better quality of life while preserving their privacy. A SH system is usually equipped with a collection of inter-related software and hardware components to monitor the living space by capturing the behaviour of the resident and understanding his activities. By doing so the system can inform about risky situations and take actions on behalf of the resident to his satisfaction. The present survey will address technologies and analysis methods and bring examples of the state of the art research studies in order to provide background for the research community. In particular, the survey will expose infrastructure technologies such as sensors and communication platforms along with artificial intelligence techniques used for modeling and recognizing activities. A brief overview of approaches used to develop Human-Computer (HC) interfaces for SH systems is given. The survey also highlights the challenges and research trends in this area.
\end{abstract}

Keywords Smart Homes - Assisted living - Healthcare - Ambient intelligence - Activity Recognition . Human interfaces · Artificial intelligence $\cdot$ Sensors

M. Amiribesheli · A. Bouchachia

Department of Computing \& Informatics

Faculty of Science and Technology

Bournemouth University, UK

E-mail: \{mamiribesheli, abouchachia\}@bournemouth.ac.uk

A. Benmansour

Department of Electrical \& Electronic Engineering

Faculty of Technology, University of Tlemcen, Algeria

E-mail: benmansour_asma@hotmail.fr

\section{Introduction}

Rapid growth of the aged population has caused an immense increase in demand for personal care, particularly for people with chronic conditions such as dementia [35]. Thus care costs continue to rise putting healthcare systems under financial pressure [120]. A relatively efficient solution to decrease these costs is to shift the emphasis from formal care in hospitals and care homes to informal care in private homes. Studies [117] revealed that most people needing care prefer less intrusive informal care. The general perception is that informal care at home is inexpensive. However, according to statistical reports [120], conventional informal care for many chronic conditions (e.g., dementia) is not necessarily cheaper than other types of care provided in hospitals or care homes.

Smart Home (SH) technology aims to support people to have a better quality of life and to ensure elderly to live comfortably and independently [30]. The SH technology is considered as a way to reduce living and care costs and to improve the quality of life for people with care needs. It has been applied for many purposes [76] like energy saving, security and safety, fall detection, light management, smoke and fire detection etc. using various solutions such as video monitoring, alarms, smart planners and calendars, reminders, etc. Equipped with sensors, actuators and eventually cameras to collect different types of data about the home and the residents, SHs can enable automatic systems or caregivers to control the environment on behalf of the residents, predict their actions and track their health condition.

A SH system incorporates different components structured in layered architecture as illustrated in Figure 1. Each layer of the system has it own function and comes with its own challenges to be dealt with. Data is collec- 


\begin{tabular}{ll|} 
Interface Layer & $\begin{array}{l}\text { - Caregivers and residents } \\
\text { interfaces }\end{array}$ \\
Data Processing Layer & $\begin{array}{l}\text { - Machine Learning ontology } \\
\text { - Data storage and presentation }\end{array}$ \\
\hline Communication Layer & $\begin{array}{l}\text { - Wireless/Wired sensor } \\
\text { networks. }\end{array}$ \\
\hline Physical Layer & $\begin{array}{l}\text { - Residents, environment and } \\
\text { physiological sensors }\end{array}$ \\
\hline
\end{tabular}

Figure 1 The layered architecture of an SH

ted as the physical layer by sensors, transmitted through the communication layer to the processing unit in the processing layer where it is analysed for activity recognition and behaviour patterns discovery. The outcome of the analysis in the form of specific information, alerts or warnings may be communicated through the interface layer to various stakeholders (resident, caregivers, resident's relatives).

There exist some research surveys devoted to SHs and ambient intelligence $[2,21,99]$. The present survey goes beyond these by (1) presenting the state of art studies at all levels: hardware, networking, knowledge engineering and reasoning, human-computer interface; (2) focusing as much as possible on studies related to healthcare; and (3) ensuring an up-to-date and full coverage of techniques, technologies and methods. We believe that the present paper provides an insightful overview of the existing work, refreshes the knowledge in this area and provides a different organisation of the material and coverage.

This paper surveys the state of the art of SHs considering the infrastructure and communication (Section 2), data processing (Section 3) and $\mathrm{HC}$ interfaces (Section 4) and discusses the challenges facing this technology before becoming mature for successful deployment (Section 5).

\section{Sensing and Networking Technologies}

An SH system consists of two types of components: hardware components and software components. The former integrates sensors and associated equipments like controllers and Gateway equipments into a single network. Sensors are often seamlessly integrated in the living space and attached to the network using either wired or wireless connection. Ultimately sensors can be remotely monitored and controlled via the Internet. Many communication technologies and protocols such as Bluetooth, ZigBee and PLC have been used in SHs. In this section we review the sensor technologies as

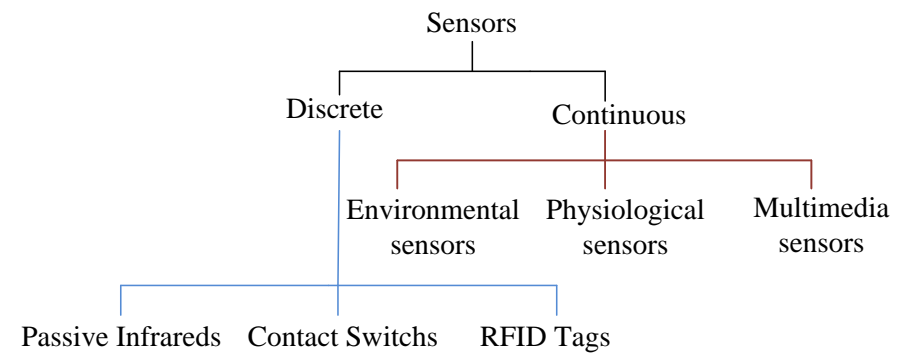

Figure 2 Taxonomic classification of sensors discussed in this paper

well as the communication networks deployed for SHs in general.

\subsection{Sensors}

Sensors are devices for detecting changes in the environment including the residents. There is a large variety of sensors used to monitor SHs and the residents. Sensors are used to collect various types of data related to [83]:

- activities of the residents

- states of the objects

- states of the environment

In particular sensors capture the following data [34, 125]:

- strain and pressure

- position, direction, distance and motion

- light, radiation, temperature and humidity

- type of material (e.g., solid, liquid and gas)

- sound

- image and video

- state of the object (e.g., present, not present)

- physiological measurements (e.g., blood sugar, blood pressure)

Sensors can be classified according to different characteristics. In the following we will categorize them based on the type of data they produce (see Fig. 2): discrete state sensors (called also "binary"), and continuous state sensors. Sensors usually form the building blocks of sensor networks. They can be either wireless or wired.

\subsubsection{Discrete State Sensors}

The output of state discrete state sensors is binary $\{0$, $1\}$, hence the name "binary" sensors. Many studies have used binary sensors for detecting the state of objects or residents (i.e. open door/closed door, light on/off, 


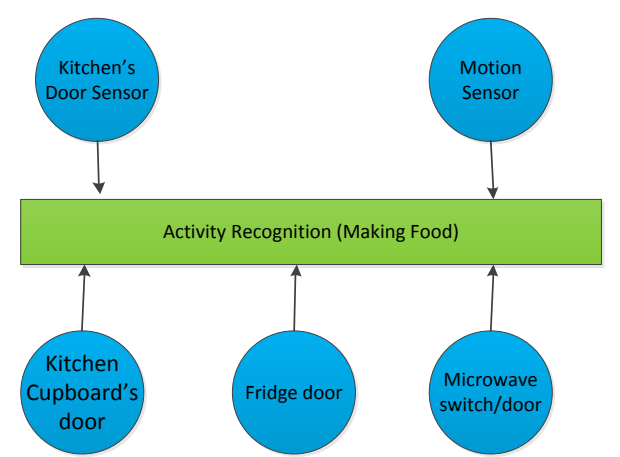

Figure 3 The use of binary sensors to capture the activity of "making food".

person movement/stillness). Due to the simple nature of the data captured and the unobtrusiveness of binary sensors, many researchers have used them to collect data about residents' activities of daily living.

The most commonly used state sensors in SHs are the Passive Infrared Sensors (PIRs) [37,70]. According to [91], all of the "effective" or promising research studies on SH have involved PIR sensors for data collection. The AILISA platform [65] utilized PIR sensors to monitor the activities of elderly people. SH systems collect PIR's readings and store them in a database using the following format: Detection [Date] [Hour] [Sensor Number]. Such data is then analysed in order to recognize abnormalities. In a study related to dementia patients, the authors in [33] used PIRs to collect data about the residents movements in the home to make sure that they are safe.

Another group of commonly used state sensors is Contact Switch Sensors (CSSs). They are used to detect the state of objects, e.g. cupboard doors and fridge doors. A typical application of CSSs to track activities can be found in [13]. Pressure sensors as part of CSS family are used to detect the presence or occupancy of spaces like a bed, chair, floor, etc. Radio-Frequency Identification (RFID) can be considered as a state technology as it serves to identify objects and people [58] using tags standing for identifiers and which are in general binary or hexadecimal sequences.

Figure 3 shows how binary sensors can be used to collect data about activities. While processing the binary data is moderately straightforward, the interpretation and presentation of binary data often require extra knowledge of the environment in order to capture the full activity. For instance, it is difficult to understand if a resident truly took his medication only by checking the captured data from the CSS attached to a drug cabinet door. Usually many sensors are required to capture the context and hence efficiently track the person.
Departing from this idea, Wilson and Atkeson [118] tried to capture the activity context by associating the resident's location and activities. Binary sensors were used including motion detectors, break-beam sensors, pressure mats, contact switches and RFID. The goal of the study was to identify the residents and track their activities in order to detect abnormal behavior.

\subsubsection{Continuous State Sensors}

In contrast to binary sensors, the output of continuous state sensors can take simple or complex forms like real numbers, images and sound. Popular sensors in this class are environmental sensors that are used to capture environmental data such as temperature, humidity, light, pressure, noise, etc. Such sensors are used to monitor all sorts of environments for different applications. Physiological sensors are used to monitor the resident's health condition. They capture different physiological data, such as blood glucose, blood pressure, ECG, EEG, EMG, pulse and body temperature. Usually physiological sensors are worn by patients forming the so-called body area network (BAN).

Continuous state sensors have been used to monitor SHs. For instance, Wood et al. developed a system called AlarmNet [122] for assisted living and monitoring. It is based on body area networks to collect physiological data from residents. AlarmNet supports different types of wireless sensors (e.g., heart rate, ECG pulse oximeter, weight, motion, dust, light, optical tripwires, CSSs) and interfaces to perform activity recognition. mPHASiS [64] is a healthcare information system that uses BAN to collect physiological data. mPHASiS monitors ECG, blood pressure, acceleration and temperature. It was designed as an end to end health care monitoring solution. In particular it triggers alert messages to inform the caregivers about specific health situations. The caregivers have access to the data of residents through a smart phone. Tia et al. [107] used lightweight BANs for patient triage in hospitals. Each patient wears a BAN that consists of a pulse oximeter, an electrocardiogram, blood pressure meter and a cough monitoring device to collect data. This study targets accident and disaster situations, but could be extended to care emergency in general.

The last type of continuous sensors is multimedia sensors that correspond to video cameras and microphones. Video cameras have been used to monitor certain situations, for instance, when the patient needs close monitoring by the caregivers. However their use in SHs has been often criticised and rejected due to privacy constraints [14]. People do not admit being watched; 
yet many researchers believe that video and audio sensors suggest that LPW technologies will shape the future of can considerably increase the accuracy of SH systems [123]. SH networking, particularly in the context of health-

Reidel et al. [94] proposed an activity recognition system based on audiovisual data. They investigated activities such as having snacks while watching TV and reading the newspaper. The data was collected from six single healthy adults using multiple cameras in the home. The study achieved in average more than $90 \%$ activity recognition accuracy. Silva et al. [29] developed another system using multimedia sensors to recognise activities like falling, walking, standing and shouting. The activity data was collected by simulation in a lab. For processing, two activity recognition models were applied, a model for processing obtained audio data and another for processing captured video data. The overall accuracies for activity recognition models were $94.44 \%$ for video data and $83.35 \%$ for audio data. Another system, called $\mathrm{COACH}$, was proposed in [75] for assisting elderly with dementia through the process of washing hands. $\mathrm{COACH}$ uses video frames to discover the hand position relying on the partially observable Markov decision processing model (POMDP). The system provides also multimedia guide and alerts the caregivers when the person is facing a risky situation (e.g., when the person is not moving, the sink is full).

Table 1 shows some of the research studies where various types of sensors for different tasks were deployed.

\subsection{Communication}

The communication layer (see Fig. 1) in SHs plays the crucial role of connecting all of the components such as sensors, actuators, gateway and storage hardware. We can distinguish the following communication technologies:

1. Low Powered Wireless (LPW) networks (e.g., zigbee, bluetooth, RFID)

2. Power Line Communication (PLC) and heterogeneous PLC standard (e.g., X10)

3. Personal computer networking protocols (e.g., WIFI)

4. Universal Mobile Telecommunications System (UMTS)

In the following a short presentation of these technologies is given.

\subsubsection{Low-Powered Wireless Networks}

Low-Powered Wireless (LPW) or low energy wireless standards have been designed in a way to allow power sensitive devices like sensors to function with minimum low consumption of energy [102] by staying in the power saving mode as long as possible. Some research studies described in the following.

ZigBee is the mostly used standard among the LPW family. It is a small, low-cost, low-power, short-range wireless technology. It operates on a signal range of $2.4 \mathrm{GHz}$ with data rate of $250 \mathrm{Kbps}$ [7]. Its transmission distance can spread up to 75 meters depending on the environment and the type of sensors deployed. Zigbee has been used in smart homes since long time. For instance, Cavallo et al. [19] used ZigBee to develop a system called "Pervasive Intelligence System for Rehabilitation and Assistance" (PISRA) dedicated to people with dementia. The system serves to track people activities within SHs such as sleep, movement, fall, social communication and taking drugs. Moreover, the PISRA system offers help and guidance to the residents for conducting their daily activities. Van Hoof et al. [110] developed a ZigBee-based system, called "Unattended Autonomous Surveillance (UAS)", to monitor the security and safety of old people with mild dementia. UAS consists of a number of wireless sensors located in the living room, bedroom and the kitchen which communicate through ZigBee. UAS detects incidents such as falling and inactivity and triggers adequate alarms when the person is in a risky situation.

U-Health [66] is a ZigBee-based system designed for monitoring older adults health using 12 different types of wireless sensors for capturing heartbeat, blood pressure, body temperature, motion, location, blood sugar, cholesterol, $\mathrm{SpO} 2$, dehydration, camera, humidity, smoke, and temperature. In the evaluation, authors only tested two of U-health sensors (the blood glucometer sensors, the ECG monitoring system) for 29 patients. They provided 20 patients with a ZigBee-based blood glucometer sensors that transmit the readings through a mobile phone to web, and caregivers were instructed on using a web service which could illustrate the readings. The other 9 have participated in ECG monitoring system, with a ZigBee-based ECG attached to their chest that transmit the readings through a mobile phone to web, and caregivers who could see the reading through web. The result showed the satisfaction scores of 8.59 and 9.01 out of ten points for blood glucometer sensors and web service, respectively. The mean satisfaction scores for ECG sensor and ECG monitoring services were 5.79 and 7.29 , respectively.

Likewise, in [104] a monitoring system relying on ZigBee was developed to track the activities of people in an SH. Authors employed a variety of sensors including pressure and contact switches attached to household appliances like microwave, kettle, toaster, heater, TV 
Table 1 Some of SHs research studies and their sensor technologies.

\begin{tabular}{|c|c|c|c|}
\hline Refrence & Sensors & Activities & Purpose \\
\hline$[124]$ & $\begin{array}{l}\text { Video cameras, } \\
\text { phones, } \quad \text { Ficro- } \\
\text { Motion, RFIDs }\end{array}$ & $\begin{array}{l}\text { Watching TV, Cooking, Tracking } \\
\text { personal items }\end{array}$ & $\begin{array}{l}\text { Behaviour monitoring, Tracking } \\
\text { personal items }\end{array}$ \\
\hline [85] & Air pressure & Not mentioned & Residents' location \\
\hline [89] & $\begin{array}{l}\text { Video cameras, Bed pressure, } \\
\text { Stove door CSS, Motion }\end{array}$ & $\begin{array}{l}\text { Cooking, Sleeping, Walking in the } \\
\text { house }\end{array}$ & $\begin{array}{l}\text { Resting hours, Behaviour monit- } \\
\text { oring }\end{array}$ \\
\hline$[116]$ & $\begin{array}{l}\text { Signal strength of wireless } \\
\text { devices }\end{array}$ & Not mentioned & Resident's location \\
\hline$[119]$ & $\begin{array}{l}\text { Motion detectors, Pressure } \\
\text { mats, CSSs, RFIDs }\end{array}$ & $\begin{array}{l}\text { Eating, Bathing, Dressing, Toileting, } \\
\text { Cooking, Watching TV }\end{array}$ & $\begin{array}{l}\text { Resident's location, Behaviour } \\
\text { monitoring and prediction }\end{array}$ \\
\hline [6] & $\begin{array}{l}\text { Accelerometer, Blood pres- } \\
\text { sure readings, Microphones, } \\
\text { Heart rate, Temperature }\end{array}$ & $\begin{array}{l}\text { Movement, Blood pressure changes, } \\
\text { Speech, Sound }\end{array}$ & Healthcare Monitoring \\
\hline [55] & 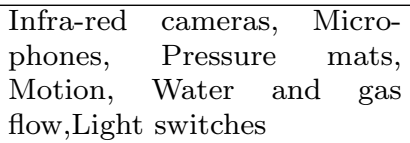 & $\begin{array}{l}\text { Cooking, Socializing, Sleeping, } \\
\text { Cleaning, Relaxing, Working }\end{array}$ & Behaviour monitoring \\
\hline$[80]$ & Motion & Not Applicable & Producing elderly's life scenario \\
\hline [94] & Video Cameras & $\begin{array}{l}\text { Getting home and watching TV, } \\
\text { Eating while watching TV, Reading }\end{array}$ & Behaviour monitoring \\
\hline [65] & Motion, CSSs & Bathing, Dressing, Toileting, Eating & Behaviour monitoring \\
\hline$[122]$ & $\begin{array}{l}\text { Heart rate, Movements, } \\
\text { ECG, Pulse oximeter, } \\
\text { Weight, Pulse monitoring }\end{array}$ & $\begin{array}{l}\text { Toileting, Sleeping, Showering, Eat- } \\
\text { ing and drinking, Walking, }\end{array}$ & $\begin{array}{l}\text { Healthcare Monitoring, Beha- } \\
\text { viour Monitoring }\end{array}$ \\
\hline [25] & Motion, CSSs & $\begin{array}{l}\text { Bathing, Walking, Cooking, Eating, } \\
\text { Relaxing, Personal hygiene, Sleep- } \\
\text { ing, Taking medicine }\end{array}$ & Behaviour monitoring \\
\hline$[115]$ & Motion, CSSs & $\begin{array}{l}\text { Toileting, Showering, Eating and } \\
\text { drinking, Walking, }\end{array}$ & Behaviour monitoring \\
\hline
\end{tabular}

and dishwasher. The system was used to estimate the well-being of the residents based on usage frequency of the appliances. The proposed system was experimented on four SHs inhabited by single old persons. The system outcome showed the type appliance and excess used by the elderly at their houses and also its outcome could be used to predict early risky situations.

The Bluetooth is a low-cost wireless communication protocol that has been originally manufactured to connect mobile and handheld devices at a maximum data rate of $1 \mathrm{Mbps}$ within up to 10 meters of distance [9]. It uses the standard $2.4 \mathrm{GHz}$ signal band for communication. However the Bluetooth technology has not been frequently used as a communication standard for $\mathrm{SHs}$, although some authors [31] claimed that Bluetooth is better because it enables users to interact with the system via conventional handheld devices such as mobile phones and tablets.

In [67], it was shown that Bluetooth supports fewer cell nodes compared to ZigBee and Wi-Fi. Moreover it has lower data rate compared to Wi-Fi. For these reasons and because also of the predominance of $\mathrm{Wi}$ Fi-enabled handheld devices Bluetooth is less perefrred for SHs.
$R F I D$ is a technology for automatic identification of the objects and people by computer-based systems. RFID tags and readers use a variety of frequency ranges that can be categorised as low (124-135 kHz), high $(13.56 \mathrm{MHz})$ and ultra-high $(860-950 \mathrm{MHz})$. As the frequency range increases the reading range increase too. The number of $\mathrm{SH}$ projects for health that utilized the RFID technology have increased significantly in the past few years. RFID was particularly used for identifying people in a multi-occupant $\mathrm{SH}$ (see Section 5). For instance, in [123] the authors used two separate RFID systems, an active RFID for environment monitoring and a passive one for resident identification (known also as data association problem).

\subsubsection{Power Line Communication}

Power line communication (PLC) technologies allow SHs to adopt universally available electrical communication terminals as the communication infrastructure. In the following, some of the most common PLC technologies are presented.

$X 10$ is an international networking protocol that enables home appliances to transmit digital data through an electrical power line. X10 is commonly recognized as a low-cost data transmission protocol with no installa- 
tion requirements as it is uses existing wiring. Although the implementation of PLC is not as flexible as the wireless technologies [3], many researchers considered X10 as a cheap and available option to control appliances. They advise that a mix of wired and wireless technologies can offer the best of the two worlds to meet the requirements of $\mathrm{SH}$ systems [24].

In [89], an X10 sensor network for monitoring old people living in a retirement community was applied. Motion sensors and pressures sensors were attached to rooms and beds. The research investigated the correlation between resident's daily activities and health problems such as falling or emergency medical needs. The study illustrated that the residents go into a period of restlessness before facing a problem.

Other Power Line Communication Technologies A number of different PLC-based protocols for home automation have been proposed. Among others, these include European Installation Bus (EIB), Home Plug and Lonworks. Often these PLC protocols are enhanced versions of X10 [48]. The Home Plug Power Line Alliance introduced a PLC network protocol which connects nodes with significant bandwidth of $200 \mathrm{Mbps}$ in an indoor distance of up to 350 meters using existing electric line [126]. In [127], the authors, discussed some reliability measures to ensure that Home Plug protocol is secure when handling sensitive data such as healthcare data. They compared home plug against some other more conventional wired and wireless protocols like 100BaseT and 802.11b. The comparison studied the network reliability factors such as the number of supported simultaneous connections, packet drops, data jitters and delays. The study stated that the PLC network successfully delivered low, medium and high bit rate data without any packet drops.

Despite the significant improvement of service in the new PLC protocols, the number of wired SH studies is limited compared to SHs that employ wireless and computer network technologies [2]. The characteristics of wireless systems like mobility, accessibility and compatibility push most of the research studies to use wireless protocols or a mix of wireless and wired protocols.

Heterogeneous networking protocols for intelligent buildings Diversity in sensor types and communication protocols have led to the development of hybrid protocols. One of the most successful heterogeneous protocols used for smart buildings is KNX [108]. KNX is a modern standard which has incorporated three European standards (BatiBUS, EIB, KNX-RF) and internet protocol to offer one package solution to $\mathrm{SH}$ networking. KNX allows designers to use various types of media such as radio frequency, power line and twisted pairs and the IP protocol. Heterogeneous protocols are not only limited to combining wired and wireless technologies, but also protocols from the same family such as home wireless (e.g. Wi-Fi) and low powered wireless technologies (e.g. ZigBee) [116].

\subsubsection{Personal computer networking protocols}

Single-board advance computing units (e.g. Raspberry $\mathrm{Pi}$, Arduino) are cheap and broadly available nowadays. Many studies tend to use computer networking protocols for SHs. Wi-Fi (Wireless Fidelity) is a computer networking protocol that is known as the IEEE 802.11 networking standard. Designers initially built it for the wireless local area networking, and it works on 2.4, 3.6 and $5 \mathrm{GHz}$ frequency bands. Until recently Wi-Fi was considered not suitable for sensor networks and exclusive for PC networking [39]. Nowadays, there is a variety of Wi-Fi enabled devices with sensors (e.g., smart phones, smart TVs, etc.) and houses are equipped with $\mathrm{Wi}-\mathrm{Fi}$ access points for internet sharing. Hence, the creation of a Wi-Fi based sensor networks for SHs will be easy as illustrated with the CareNet [57].

\subsubsection{Mobile Telecommunications System (MTS)}

MTSs are capable of transmitting different types of data such as text, digitized voice, images, and video. MTS can be used in SH applications. For instance studies in $[42,109,129]$ adopted the SMS as an instrument for interacting with SHs' monitoring and remote control applications. In [4], the authors proposed a control system for $\mathrm{SHs}$ that allows the users to manage home appliances (e.g. air conditioner, light) by SMSs. The same platform could be applied to $\mathrm{SH}$ in healthcare. Airmed-cardio [100] is an MTS based monitoring system for cardiac patients follow-up in their home. Each patient has a portable monitoring equipment and cellular phone that supports data transmission. The collected data is transmitted to a base station which is monitored by a human operator.

\section{Data Processing and Knowledge Engineering}

Following the layered architecture presented in Fig. 1, the data is collected and transmitted through the communication medium to a data processor. In this stage, data potentially undergoes a pre-processing step for cleansing and preparation before further processing is initiated. The main step is, however, analysis which encompasses: mining behavioral patterns, recognizing activities, detecting abnormal behaviour, etc. This stage is the "smart part" of a $\mathrm{SH}$ system. 
For healthcare application of SHs, the aim of data processing is to:

- present the sensor data in a way that caregivers can track the changes of the resident's health state and how the daily activities are accomplished by the resident under observation.

- detect anomalies when carrying on activities and trigger alerts in critical situations (i.e. falling down, forgetting to turn off the cooker).

- identify the progress of chronic diseases and conditions (e.g. case of elderly with dementia).

- remind the resident about scheduled activities (e.g. taking pills).

- predict activities by the resident and assist him in their accomplishment.

A variety of knowledge engineering and data processing methods can be used to analyse the collected data. In the following we will focus on the main problem in SHs which is activity modelling and recognition from sensor readings. The common computational models used for activity recognition in SHs will be highlighted and related studies will be summarized. The models considered are: decision trees, fuzzy logic, artificial neural networks, support vector machines, Naive Bayes classifier, hidden Markov models, emerging patterns, and ontologies. Table 2 shows some of the SH research studies including the data source, the algorithms, and the performance of such algorithms.

\subsection{Decision Trees}

A decision tree (DT) is used to model the relation between input data and the corresponding output. A decision tree can be used for either classification if the output is discrete indicating class labels or regression if the output is continuous. A classification tree consists of nodes that represent features and branches that represent the values of the features. The leaf nodes represent the class labels. Figure 4 shows a classification tree with 2 classes: safe and risky. A tree can be rewritten as a set of IF-THEN rules. For instance the rule: IF Resident_in_kitchen $=$ NO and Gas_Hobs_Timer $>30$ THEN Class=Risky

can be derived from the tree in Fig. 4. DTs are built through an induction process using a training dataset. Many induction algorithms have been devised such as TDIDT/ID3, C4.5, CART, MARS, and CHAID. Some algorithms like C4.5 and CART execute two phases: growing and pruning of the tree, while others only grow the tree [98].

In [56], C4.5 was used to generate a DT for classifying the actions of the resident that is a combination

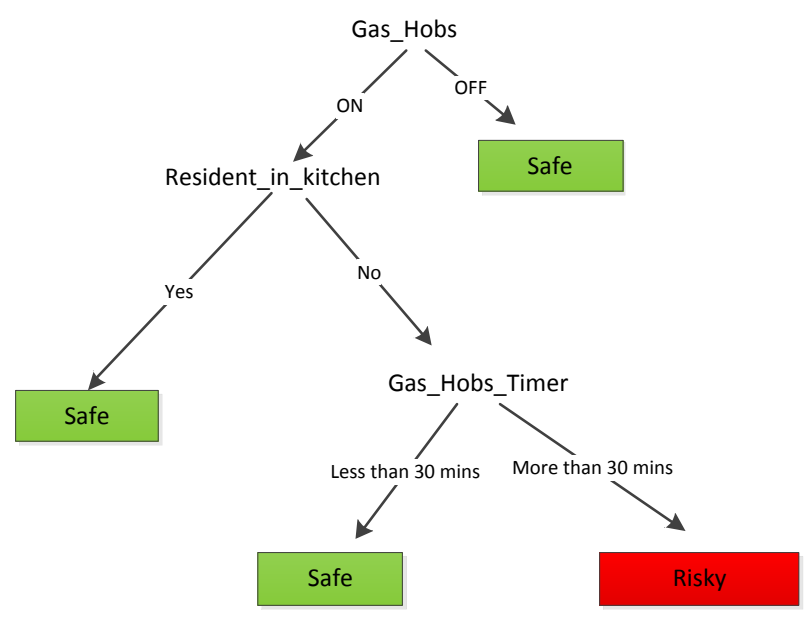

Figure 4 The structure of a DT.

of the resident location and the object touched. The training dataset was collected from pressure sensors on the floor for locating the resident and RFID tags on the objects (e.g. gas hobs, cupboard). The evaluation of the classifier on kitchen activities achieved an accuracy of $90 \%$ to $100 \%$ depending on the size of learning data used.

C4.5 was also applied in [90] to recognize activities using data from a wearable triaxial accelerometer. The activities considered were: standing, walking, running, climbing up stairs, climbing down stairs, sit ups, vacuuming and brushing teeth. C4.5 was used among other classifiers such as k-nearest neighbour, SVM and Naive Bayes. The results showed that C4.5 achieved 97.29\% when trained and tested on data from the same user over many days. An accuracy of $98.53 \%$ was achieved when $\mathrm{C} 4.5$ was trained and tested on data stemming from many users and over many days and $77.95 \%$ when trained and tested on data not from the same day.

In [72], the authors used ID3, the perceptron and $\mathrm{k}-\mathrm{NN}$ to determine the location of the resident based on the wireless signal strength. Two datasets were used: Peter Kiewit Institute dataset (PKI) and Maxwell Working (MD) dataset [88]. The results showed that for ID3, the mean error was $4.9 \mathrm{~m}$ and $2.5 \mathrm{~m}$ for PKI and MD respectively. The mean error of k-NN was $4.9 \mathrm{~m}$ and $2.4 \mathrm{~m}$, while that of the perceptron was $7 \mathrm{~m}$ and $2.4 \mathrm{~m}$ respectively.

In [87], the authors applied decision trees to model activities of daily living in a multi-resident context. An extension of ID5R, called E-ID5R, was proposed where the leaf nodes are multi-labeled. E-ID5R induces a decision tree incrementally to accommodate new instances and new activities as they become available over time. To evaluate the proposed algorithm, the ARAS dataset 


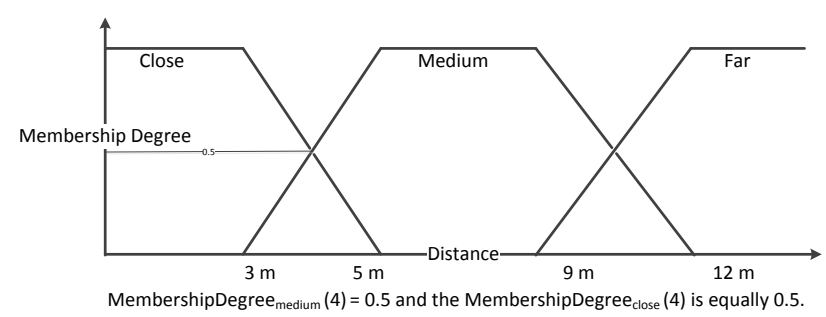

Figure 5 Fuzzy set represented as a fuzzy membership function.

which is a real-world multi-resident dataset stemming from two houses was used. E-ID5R performs differently on activities of both houses: for house $\mathrm{A}$ whose data is quite challenging, the classification rate was modest (40\%), while for house B the rate approached $82 \%$.

\subsection{Fuzzy Logic}

As an extension of the classical set theory, Zadeh [128] defined a fuzzy set as "a class of objects with a continuum grades of membership. Such a set is characterized by a membership (characteristic) function which assigns each object to a grade of membership ranging between zero and one. Fuzzy sets have been used to develop fuzzy logic systems. Many SH studies have applied fuzzy logic to build monitoring and prediction systems.

To explain what is a fuzzy set, the primary step is to understand the membership function. This function maps each input data to a membership degree $\in[0,1]$. For instance, Fig. 5 illustrates membership value of the distance value 4 to three to three fuzzy sets corresponding to the linguistic concepts: "close", "medium" and "far".

Using fuzzy sets, rule based systems (RBS)can be extended to include fuzzy "IF-THEN" rules of the form: IF sink_water_level is High then stop_tap. As shown in Fig. 6, a fuzzy RBS has the same components as a traditional RBS but the input is fuzzified at beginning and the output is defuzzified at the end. The inference system maps the input data to the rule base and aggregate fuzzy output of the system according to an inference method. The defuzzification converts the fuzzy output of the system to crisp output.

The fuzzy rule-based systems are used in the form of fuzzy rule-based classifiers. For instance, a fuzzy rule looks as follows: IF Resident_stay_in_bed for Long and bedroom_TV=OFF then Class_risky. Rules can be associated with confidence level which may be obtained in different ways. The structure of a fuzzy rule-based classifier is similar to the Figure 6 however since the outputs are classes the defuzzification step is often not

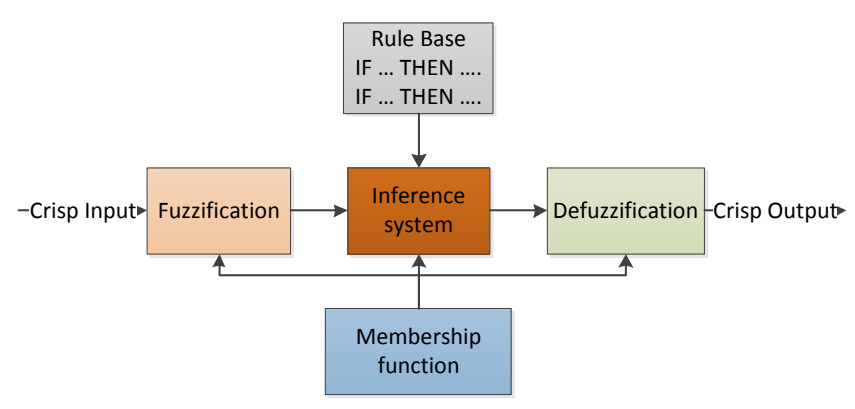

Figure 6 Structure of a fuzzy rule-based system

necessary. A good overview of fuzzy classifiers can be found in [11].

In [46], the authors used a fuzzy rule-based controller where the output of the rules is not a class but a function in order to monitor a smart environment, called "iDorm", based on preferences of the occupants. The data was generated over two months by recording two student activities in a dormitory flat which is equipped with 11 sensors: internal light level, external light level, internal temperature, external temperature, chair pressure, bed pressure, occupancy and time etc. The output is provided by six actuators: variable intensity spot lights, the desk and bed side lamps, the window blinds, the heater and PC-based applications comprising of a word processing program and a media playing program. The induction process led to a rule base consisting of 280 rules based on 132 hours of collected data.

Bouchachia [10] introduced a rule-based fuzzy classier, called IFS that stands for incremental fuzzy classification system, which is capable of dealing with the dynamic nature of SHs. The classifier is based on generalized fuzzy minmax neural networks (GFMMNN). It is designed to learn and self-adjust in a exible manner to react to dynamic changes such as the occurrence of new events, inputs' change, seasonal changes, etc. The study demonstrated how fuzzy rules can be continuously generated online to meet the requirements of a dynamic environment. The author used the iDorm data [46]. The data was split into two subsets: the training set contains $75 \%$ samples for the first month, and The testing set contains $25 \%$ of the first month and all samples of the second month. The evaluation of IFS shows that the adaptation is essential for online tracking of people's activities. The current error rate continuously decreases reaching 0.01. Compared with fuzzy ARTMAP and nearest generalized exemplar, IFS produces a lower current error.

In [81] some fuzzy classifiers such as Class0, eClass1, k-NN, NB and HMM were used to recognize activities. The following activities were considered: leaving 
the house, using the toilet, showering, sleeping, eating breakfast, eating dinner and drinking. Using data collected from 3 smart homes, it was found that on average the evolving classifiers, eClass0 and eClass1 achieve an f-measure value of 0.6 and 0.7 respectively outperforming the other classifiers, especially when the size of the training data increases.

Bouchachia and Vanaret [12] developed an online type 2 self-learning fuzzy classifier, called GT2FC standing for Growing Type-2 Fuzzy Classifier. For the rule learning from data streams. They suggested that the classifier is suitable for ambient intelligence (e.g. SHs) where the goal is to use sensed data to monitor the living space on behalf of the residents. The research tackled three challenges of online learning, complexity of the rule-based classifiers and accommodation of labelled and unlabelled data during rule learning. To illustrate the model's performance, they conducted experiments using an ambient intelligence which iDorm dataset [46]. The paper investigates the effect of labelled and unlabelled on the classification accuracy. It also compares the outcome of GT2FC against other online classifiers such as IFCS [10], Nearest Generalized Exemplar, Growing Type-1 Fuzzy Classifier with 70\% of data being labelled, the accuracy of GT2FC classifier was $81.65 \%$ while the GT1FC was 81.42 , Nearest Generalized Exemplar was $75.54 \%$ and IFCS was $75.24 \%$.

\subsection{Artificial Neural Networks}

Artificial Neural Networks (ANN) is a computing model made up of a number of simple, highly interconnected processing elements, which process information by their dynamic state response to external inputs [18]. The fundamental processing elements of an ANN are articial neurons (or nodes) which are interconnected by weighted links forming layers as shown in Fig. 7. Typically in an ANN there is one input layer and one output layer and a number of hidden layers that varies depending on the complexity of the problem at hand [79]. Neurons transform the weighted input into output using an activation function which can take different forms (linear and non-linear). The process by which the weights are adjusted is called learning. A number of non-linear ANNs are known to perform as function approximators. There are various parameters that define the architecture of a neural network: the connection type (e.g. feed-forward networks recurrent neural networks etc.), learning rule (e.g. hebbian rule, perceptron learning, back-propagation, etc.), and activation functions (e.g. sigmoidal, hyperbolic tangent, etc.). Because of these shaping parameters, there are different types of ANNs

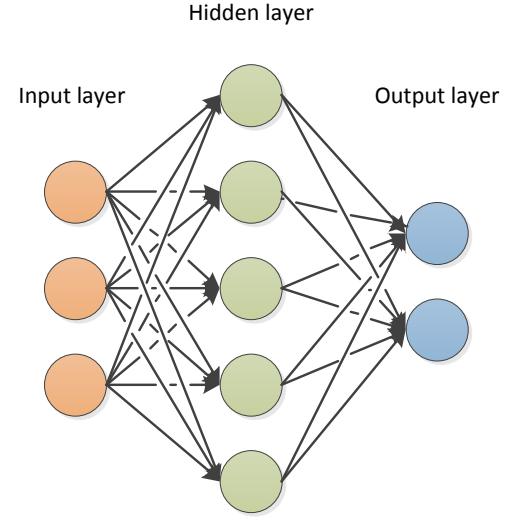

Figure 7 Architecture of a 3-layer feedforward network.

(e.g. Multi-Layer Perceptron (MLP), Echo State Networks (ESN), Radial basis function networks (RBFN), Boltzmann machine, etc.).

ANNs can be applied to a number of $\mathrm{SH}$ problems such as activity classification, control of appliances, novelty and anomaly recognition and prediction of activities. In relation to health monitoring in $\mathrm{SHs}$, ANNs were used to diagnose and monitor chronic diseases as well to build medical decision support systems $[36,60,69]$.

MLP is the most commonly used ANN models for activity recognition [8]. A precursory work using MLP for SHs is presented in [78]. MLP is used to control the energy consumption in accordance with the lifestyle of the residents. It is trained using the back-propagation algorithm on a dateset collected from state of the lamps, intensity level of the lamps, speed of the fans, temperature, illumination, sound level, motion and state of the doors and windows. By predicting the future activities of the resident using this MLP, energy consumption of appliances in the home can be monitored.

In the MavHome project [26], an MLP-based framework was proposed to detect activity anomalies and identify repetitive tasks performed by residents. The sensors used in this framework are motion, light, humidity, and CSS. The empirical evaluation showed an average of $64 \%$ accuracy for activity recognition in five randomly generated resident ADL scenarios. RiveraIllingworth et al. [95] employed a recurrent neural network (RNN) based on Evolving Connectionist System (ECoS) [59] to recognize activities like sleeping, eating, working with computer, and to detect abnormal behaviours. ECoS operates online, which means that new sensors can be added to the architecture, and new activities can be accommodated at any stage. The authors evaluated RNN using a dataset collected from a student dormitory in Essex using different sensors like light, temperature, pressure, etc. They achieved an av- 
erage of $74.57 \%$ accuracy rate on a test dataset and $89.14 \%$ of abnormal activity detection rate.

In [68] a One-Pass Neural Network (OPNN) was applied to identify anomalies and to perform activity recognition. Similar to EcoS, OPNN runs online. In the study, a bedroom was used as an experimental space. The room was equipped with a set of sensors like chair and bed pressure sensors, light, table lamp, bed lamp, air condition, and window blind. To produce the dataset of simulated ADLs, the authors gave questionnaires to the students to annotate their activities (e.g. working on the table, computer use, listening to music, sleeping). OPNN was evaluated on a sample of achieving $92 \%$ accuracy. A layer was added to OPNN for deciding the type of abnormality: abnormal behaviour, abnormal sequence, and abnormal frequency. For instance, when the duration of cooking exceeds a specified upper limit or when using the toilet frequently, the system will perceive this as abnormality.

Lotfi et al. [70] applied Echo State Networks (ESN$\mathrm{NN}$ ) to predict future abnormal activities for elderly with dementia. The system aims at identifying anomalies such as sleep deprivation and sending alerts or reports via e-mail or phone to the caregivers. The data used was collected from PIR and CSS sensors. The empirical evaluation produced an anomaly prediction rate of $93 \%$ to $99 \%$ for very simple activities. In this study $\mathrm{k}$-means and fuzzy c-means clustering algorithms were applied to detect abnormal activities using the starttime and the duration of activities. Large clusters have found to match normal activities and smaller ones to as abnormal activities.

\subsection{Support Vector Machines (SVMs)}

Support Vector Machines (SVMs) are quite popular classification methods and have been used various applications such as face identification, text categorisation and stock classification. SVMs have been used for activity recognition in a number of studies. Consider the linearly separable data in Figure 8. Classes are separated by hyperplanes. SVMs maximize the margin around the separating hyperplane. The margin is the distance from the hyperplane to the closest data points which are called support vectors. SVMs use different optimization techniques to find the optimal hyperplane by maximising the margin. SVMs use kernel functions (e.g., radial basis kernel, polynomial kernel, etc.) to map the non-linearly separable data from the input space into a higher space where data become linearly separable.

SVMs have been used for activity recognition in a number of studies. For example, an application of SVMs was described in [40]. The data was collected through a

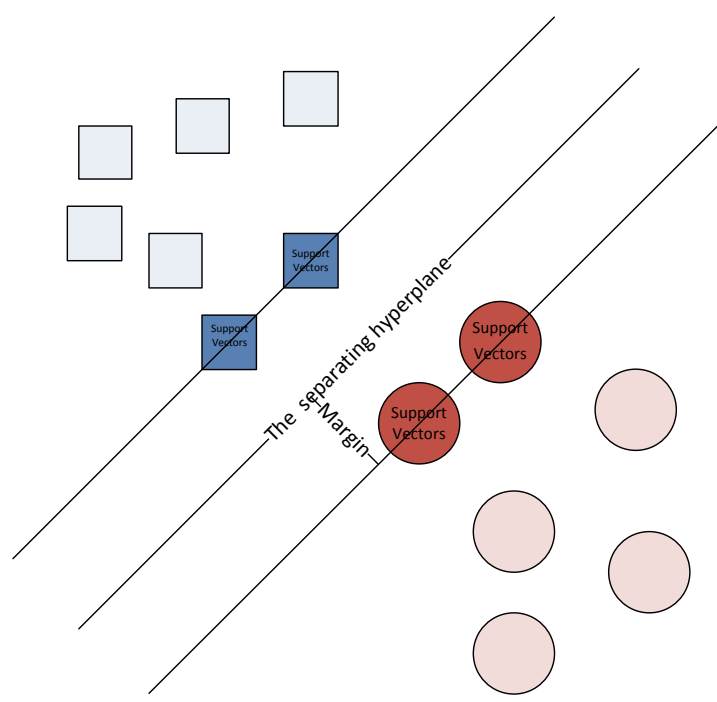

Figure $\mathbf{8}$ The hyperplane, margin and support vectors.

set of binary (e.g., PIR, CSSs, flood detectors) and nonbinary (e.g., microphones, wearable kinematic sensors) sensors. An SVM-based system was applied to recognise seven activities: sleeping, resting, dressing, eating, using the toilet, hygiene activities and communications. The cross-validation test gave the results with a satisfactory classification rate of $75 \%$ for a polynomial kernel and $86 \%$ for a Gaussian kernel.

Cook et al. [25] employed SVM and the Naive Bayesian classifier (see Sec.3.5) and Hidden Markov Model (see Sec.3.6) to deal with activity discovery and recognition. The data was obtained from $3 \mathrm{SHs}$ inhabited by elderly residents using PIR and CSS on doors. The daily activities targeted included bathing, walking, cooking, eating and taking medicine. The results revealed that SVM outperformed the other models, achieving an average accuracy of $91.52 \%$ for the 3 homes. Moreover, an activity discovery model was introduced in order to detect novel activities and to enhance activity recognition. SVM could improve the accuracy up to $10 \%$ when using activity discovery along with activity recognition.

In [71] applied SVM among other algorithms (C4.5 DT, NBC, K-nearest neighbour, and random forest) for fall detection. The data was collected using 12 radio tag equipments attached to the shoulders, elbows, wrists, hips, knees and ankles of three people. The evaluation of the algorithms showed that SVM outperforms the rest of classifiers obtaining an accuracy of $97.7 \%$.

In [49] an activity recognition technique based on the discrete cosine transform (DCA) and SVM was proposed. The dataset was collected via a Bluetooth-based triaxial accelerometer from 11 people. Four activities were considered: running, staying still, jumping and 
walking. First, the features were extracted from the data employing DCA, then SVM was trained to recognise these activities. A high accuracy of $97.51 \%$ was obtained.

\subsection{Naive Bayes Calssifier (NBC)}

The Naive Bayes classifier (NBC) is one of the simplest probabilistic classifiers. It relies on Bayes' theorem to build the decision boundary making use of the assumption that all of the input features are independent. This assumption makes the classification process tractable. Given an input $X=\left[x_{1}, x_{2}, \cdots, x_{d}\right]^{t}$ and a set of classes $\left\{C_{1}, \cdots, C_{J}\right\}$, the conditional probability $P\left(X \mid C_{j}\right)$ can be written as:

$P\left(x_{1}, x_{2}, \cdots, x_{d} \mid C_{j}\right)=\prod_{i=1}^{d} P\left(x_{i} \mid C_{j}\right)$

The label of $\mathrm{X}$ is then predicted as follows:

$C=\underset{j=1 \cdots J}{\arg \max } P\left(C_{j}\right) \prod_{i=1}^{d} P\left(x_{i} \mid C_{j}\right)$

NBCs have been employed in many $\mathrm{SH}$ research studies. Tapia et al. [106] used NBC to recognize daily activities such as washing hand, toileting, cooking, resting and dressing. A set of 77 binary sensors were installed on targeted objects like doors, windows, cabinets, a microwave, a stove and a dishwasher. Using accuracy (number of times the activities were correctly recognized) and whether the activities were detected with or without delays, NBC has produced the best accuracy of $89 \%$ considering different settings. Using the same dataset, Kasteren et al. [114] applied dynamic NBC (DNBC) for activity recognition. The experimental results showed that DNBC outperformed NBC.

NBC was also evaluated on a video data in [74]. In this study activities like "working with the phone", "drinking water" and "eating snacks" were targeted. The experimental evaluation showed that NBC could perform well on video data achieving an accuracy of $89 \%$.

\subsection{Hidden Markov Model and its Variants}

Hidden Markov Model (HMM) is a special case of Bayesian Networks which model joint probabilities of states and observations.

It used to estimate hidden state sequence $\left(y_{1}, y_{2}, \cdots, y_{T}\right.$ given the input sequence $\left(x_{1}, x_{2}, \cdots, x_{T}\right)$ as illustrated in Fig 9.

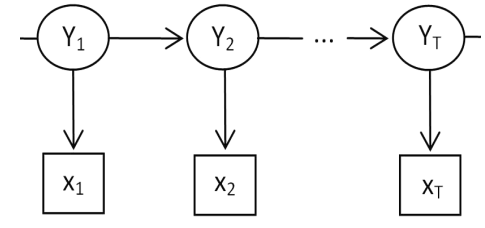

Figure 9 Structure of HMM

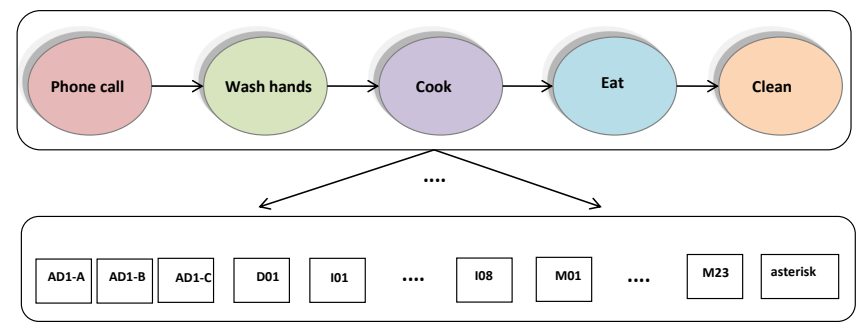

Figure 10 Activity modeling using HMM

HMM is one of the most common computational models applied to activity recognition. As shown in Fig 10, the activities represented as ovals are modeled as hidden states, while observations (called also observed states) represented as rectangles indicate the data emitted by the sensors. The horizontal edges represent transition probabilities and downwards edges represent the emission probabilities of the corresponding observed state.

This encoding was reflected on in [115] where HMM was applied to recognize some target activities: leaving the house, toileting, showering, sleeping, preparing breakfast, preparing dinner and preparing a beverage. Using a set of 14 CSSs located on doors, cupboards, refrigerator and toilet flush were used to collect data, the study investigated 3 aspects: data representation, size of the training data and difference between on-line and off-line inference. The experimental results showed that a better data representation through preprocessing improves the recognition ability of HMM, while a minimum of 12 days of data is required for an efficient training. The experiments also showed that off-line inference is more effective than on-line.

In another study [23] HMM was applied on the MIT PlaceLab [106]. Compared to many similar studies, this study focuses on tracking a whole behavior as a sequence of activities that occur close to one another in time, in one location. Thus, the activities are seen as a data stream giving room to consider variable and fixed sized windows of observations. Two accuracy measures were used: behavior-level recognition and observationlevel recognition. In the first the HMM output is compared against the ground truth whenever the behaviour changes, while in the second the output is compared against the label of the observation. Using dif- 


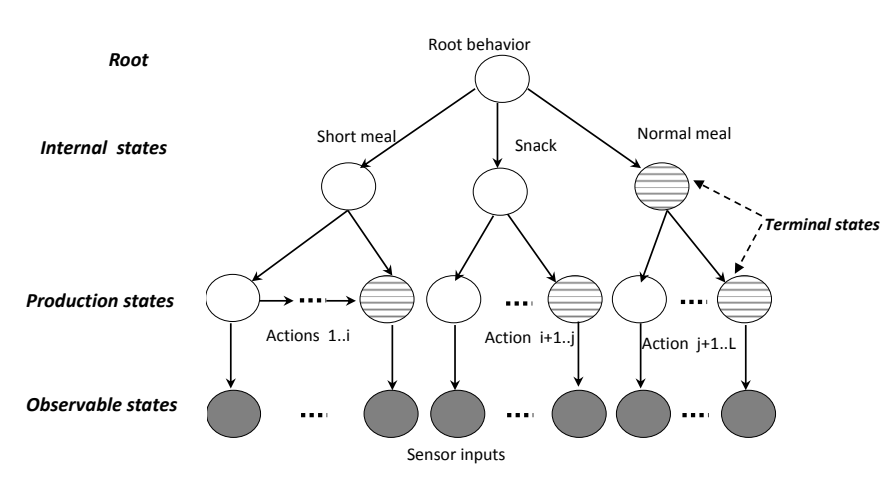

Figure 11 HHMM representation

ferent splits of data, the experiments showed that variable sized windows provided very good recognition rate using both types of measures. However, the values of the observation-level measure were always higher than those of the behavior-level measure.

Despite its popularity, HMM suffers from several limitations. HMM cannot capture long-range or transitive dependencies of the observations due to the strict independence assumptions on the observations. Furthermore, without significant training, HMM may not be able to recognize all of the possible observation sequences that lead to a particular activity [61]. Moreover HMM does not take temporal considerations into account, meaning that the duration of an action is not explicitly modeled and the interaction cannot be encoded directly. To overcome these limitations, Hidden Semi-Markov Model (HSMM) was proposed to explicitly model the duration of activities.

HSMM was compared against HMM in [112]. In this study, binary CSS sensors were used to collect 3 datasets related to two different individuals who are 26 and 57 years old. Activities that were targeted include eating main meals, using a dishwasher, drinking and eating snacks. To evaluate the accuracy of HSMM and HMM, the f-measure was used. The experimental evaluation showed that HSMM (65.5\%) outperformed HMM (54.1\%). This means that considering duration improved the classification, specifically when the sensor data does not provide enough information for activities to be distinguished.

Another variation of HMM is the Hierarchical Hidden Markov Model (HHMM). HHMM was devised to cope complex activities that can be split into smaller units (actions) with a hierarchical structure. It extends the traditional HMM in a hierarchic manner to include a hierarchy of hidden states. Each state SHMM is generalized recursively as another sub-HMM with special terminal states. Thus, HHMM contains 3 types of states (see Fig. 11): root, internal and production states. The root state is the starting node at the top of the hier-

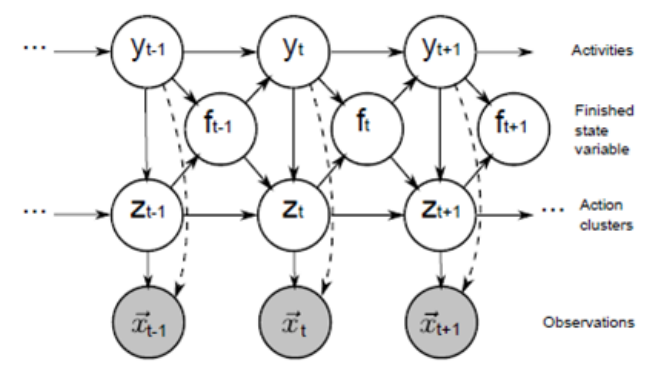

Figure 12 HHMM representation as proposed in [113]

archy. The production state is the leaf node which emits the observable output and the internal state is the composite node which is composed of several internal substates or production sub-states and does not emit observable output directly.

Van Kasteren et al. [113] investigated the application of HHMM in activity recognition following the model shown in Fig. 12. A set of sensors were used to collect the data from three houses: CSSs, pressure mats, PIRs, and toilet float sensors. It was found that when the actions are allocated separately for each HHMM outperform the other models like HMM and HSMM using the F-measure. For the first house HHMM, HMM and HSMM achieved 79\%, 72\% for HMM and HSMM. About 56\% for HHMM, 51\% for HMM and $52 \%$ for the second house, while $52 \%$ for HHMM and $45 \%$ for HMM and HSMM for the third one were obtained.

\subsection{Conditional Random Field (CRF)}

CRF is a discriminative graphical model in contrast to HMM and its variants which are generative. CRF is the most popular discriminative model and can be applied to find a hidden state transition from observation sequences. However, instead of finding a joint probability distribution $\mathrm{p}(\mathrm{x} ; \mathrm{y})$ as HMM does, CRF attempts to find only the conditional probability $\mathrm{p}(\mathrm{x} \mid \mathrm{y})$. Moreover, it allows arbitrary, non-independent relationships among the observations. Hence, it is more flexible. Another significant difference is the relaxation of the independence assumptions, in which the hidden state probabilities may depend on the past and even future observations [105]. A CRF is modelled as an undirected acyclic graph, flexible for capturing any relation between an observation variable, and a hidden state see Fig. 13.

Studies presented in $[59,112]$ have demonstrated that CRF commonly gives a better accuracy than other probabilistic models in the context of activity recognition. In the first study [59] CRF and HMM were compared using different activity data representations to show that CRF outperforms HMM. In the second study [111] CRF 


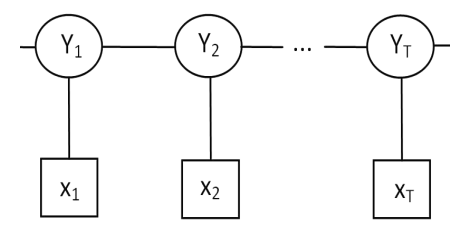

Figure 13 Structure of CRF.

was compared against NB, HMM, and HSMM and CRF scored again better.

\subsection{Emerging Patterns (EPs)}

Emerging Patterns (EPs) [45] were used to model and discriminate the activities. Thus, an EP of an activity consists of the most discriminating set of features. For instance, $\{$ location $=$ kitchen, object $=$ stove $\}$ is an EP for the activity "cooking", while \{ object = cleanser, object $=$ plate, location $=$ kitchen $\}$ is an EP for "cleaning the kitchen". The set of EPs of an activity constitute the corresponding activity model. Often the features in an EP can be found simply by frequency counting.

$\mathrm{Gu}$ et al. [45] showed how activity models can be built from sensor readings using EPs. To evaluate this technique, a real-world data set was collected from wearable sensors (RFID wristband readers and iMote2 sets) by 4 people. In all 26 common ADLs such as coffee making, tea making, oatmeal making, shaving, toileting, etc. have been considered. EPs were then generated for each activity. Using time-slice accuracy to recognize activities through intervals of time, an average accuracy of $85.84 \%$ was achieved for different activities.

\subsection{Ontological modeling}

Used to model, encode, and represent knowledge of a given domain, an ontology is a conceptual model consisting of a set of representational concepts. The concepts are typically classes, attributes, and relationships [43]. Activity ontologies describe the hierarchy of activities, activity types and their relationships. Relationships can be of different types: "is-a" or "part-of". For instance, "MakeTea" is a subclass of MakeHotDrink. Properties are used to establish the interrelations between concepts. For instance, "hasDrinkType" is a property of the "MakeHotDrink" activity that links the DrinkType concept (e.g., tea, coffee, chocolate) to the "MakeHotDrink" concept. The ontological approach to activity modelling defines the formal semantics of human activities using some ontological language like OWL and RDF.
Ontological reasoning is used to recognize activities by identifying contextual information such as sensor reading, location of persons and objects, properties of objects, etc. Chen et al. [22] described an algorithm for recognizing activities using ontologies which can be illustrated by the following scenario. For example, the activation of the contact sensors on a "cup" and "milk bottle" can link the "cup" and "milk" to the unknown activity through a concept "hasContainer" and "hasAddings" properties. By aggregating sensor observations along a time line, a specific situation that corresponds to an unknown activity could be reached. For instance the situation can be described by "hasTime(10am)", "hasLocation(kitchen)", "hasContainer(cup)" and "hasAddings (milk)". By matching this situation against activity ontologies, the activity class that mostly overlap with the situation (e.g., "MakeDrink") is considered to be the actual activity.

Riboni et al. [93] followed a similar idea using activity ontologies to recognize the daily activities. In particular they evaluated the effectiveness of the ontological approach based on dataset described in [115]. This study showed that ontological techniques underperform the data-driven techniques like HMM in absence of temporal reasoning. But when ontological techniques were extended with temporal information, their effectiveness (80.3\% accuracy) becomes comparable to HMM (79.4\% accuracy).

\subsection{Context-Aware Reasoning}

Context awareness refers to the exploitation of various kinds of information to recognise the environmental conditions within the living space to help make the smart home system intelligently interactive and selfadaptive. Information can be temporal, spatial, and related to the resident and objects in the environment [1]. Context awareness enhances the usability of a system by enabling it to react suitably and offers optimised interfaces for different contexts. There exist a number of approaches for modelling and reasoning about the context such as Unified Modelling Language, Objectoriented Models, key-Value models, and domain/web ontologies. The latter enable the system to define contexts semantically and share common knowledge of the structure of context among users, devices, and services [44].

In [50], a reference model for healthcare contextaware $\mathrm{SHs}$ was introduced. The context was generated by a set of sensors and actuators that were managed through OSGi (Open Service Gateway initiative) service bundles. System objects represented the sensors and actuators in the environment and were modeled 
along with contexts using ontologies. Authors presented two mere applications of "Smart Floor" and "Smart Plug" to prove the effectiveness of the system.

Wongpatikaseree et al. [121] introduced a contextaware activity recognition system. An ontology was exploited to model the context which is obtained through a set of sensors. To handle various activities of daily living, especially in ambiguous situations, the context is augmented with information obtained through the ontology which consists of various classes. To illustrate how the system works, some simple activities such as watching TV, relaxing and sleeping and were simulated.

Fenza et al. [38] proposed a hybrid context-aware healthcare system consisting of a web service ontology (OWL-S) and a fuzzy rule base. The residents' health context is obtained by means of sensed health data such as heartbeat, blood pressure, blood sugar, blood oxygen, height, weight, age, and temperature. The data is used to run a fuzzy rule-based clustering. Given an input, the fired rule indicates the cluster in its consequent part. The premise of the rule, corresponding to the context, is matched against the description of the service required in the current context of the person. In order to evaluate approach, a simple rule was studied using recall and precision to indicate to which extent the context is well identified through the matched services. The results obtained were moderate.

Authors in [92] introduced a hybrid of ontology and statistic context-aware activity recognition system adopting the Android platform. Activities such as walking, brushing teeth, writing on a blackboard and hiking were considered. The data was collected via a phone GPS and Accelerometer, and an accelerometer wristband. The activity recognizer relied on an ontological reasoning which is combined with statistical classification to recognize activities that are not identifiable by the classifier only. The experiments showed an activity recognition accuracy of the $93.44 \%$.

\section{Human Interfaces}

Interfaces of an $\mathrm{SH}$ must be designed in a way that empowers the users (stakeholders) to interact effectively and comfortably with the SH system. In the case of SHs for healthcare, we can distinguish four groups of users:

- Residents (e.g., dementia patients, disabled people, elderly people, etc.)

- Informal caregivers (e.g., family members of older adults)

- Social caregivers (e.g., care homes, professional caregivers)

- Formal caregivers (e.g., doctors, nurses)

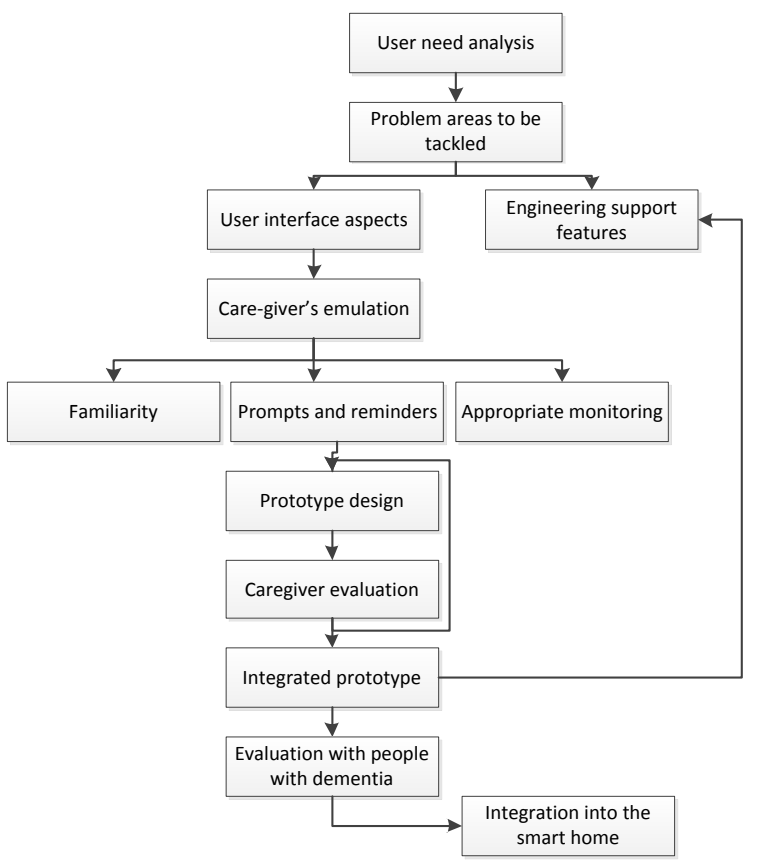

Figure 14 A Design methodology for developing SH technologies for people with dementia [82]

Therefore the design requirements of the interface must be specific for these user groups. For instance, a formal caregiver is interested in receiving updates about the progress of the resident's disorder by capturing physiological signs such as blood pressure, blood sugar and body temperature. However, such information is not necessarily relevant to the informal caregivers. Moreover, choosing an adequate interaction medium for a stakeholder needs particular considerations. As an instance, people with dementia might not be able to learn how to operate a new equipment; thus SHs for people with dementia should be able to operate regardless of the residents' capacity.

A detailed design methodology is required to acknowledge unique requirements and specifications of SHs for healthcare. The design methodology of SH interfaces follows the standards of general user interface design that consists of: requirement analysis, design, and evaluation. Sommerville [103] defined a functional user interface in a way that matches the skills, experience, and expectations of the anticipated users. Four main human factors should be taken account of during the process of designing interfaces:

- Users have limited short-term memory

- Users make mistakes

- Users are different

- Users have different interaction preferences 
Table 2 Excerpt of SHs research studies

\begin{tabular}{|c|c|c|c|c|c|}
\hline Reference & $\begin{array}{l}\text { Dataset } \\
\text { Type }\end{array}$ & Algorithm & Target & $\begin{array}{l}\text { Evaluation } \\
\text { metrics }\end{array}$ & Results \\
\hline [78] & private & ANN (MLP) & ADL(General) & Not mentioned & - \\
\hline [26] & private & ANN (MLP) & ADL(General) & Accuracy & Activity recognition: $64 \%$ \\
\hline$[95]$ & private & ANN (EcoS) & ADL(Healthcare) & Accuracy & $\begin{array}{l}\text { Anomaly detection: } 74.57 \% \text {, } \\
\text { Activity recognition: } 89.14 \%\end{array}$ \\
\hline [68] & private & ANN (OPNN) & ADL(Healthcare) & Accuracy & Activity recognition: $92 \%$ \\
\hline [70] & private & ANN (ESN) & ADL(Healthcare) & Accuracy & $\begin{array}{l}\text { Abnormally detection: } 93 \%- \\
99 \%\end{array}$ \\
\hline$[56]$ & private & DT (C4.5) & ADL(General) & Accuracy & $\begin{array}{l}\text { Activity recognition: } 90 \% \text { - } \\
100 \%\end{array}$ \\
\hline$[90]$ & private & DT (C4.5) & ADL(General) & Accuracy & $\begin{array}{l}\text { Activity recognition: } 57 \% \text { - } \\
97.29 \%\end{array}$ \\
\hline$[72]$ & private & DT (ID3) & $\begin{array}{l}\text { Resident's Loca- } \\
\text { tion }\end{array}$ & $\begin{array}{l}\text { Mean error of loc- } \\
\text { ation prediction } \\
\text { in Meters }\end{array}$ & $\begin{array}{l}\text { The Mean error: } 4.9 \text { meters } \\
\text { on the first dataset and } 2.5 \\
\text { meters on the second dataset. }\end{array}$ \\
\hline$[46]$ & private & ISL (Fuzzy) & ADL(General) & $\begin{array}{l}\text { Number of the } \\
\text { generated rules } \\
\text { by the algorithm }\end{array}$ & 280 rules in 72 hours. \\
\hline [47] & private & Fuzzy type-2 & ADL(General) & RMSE & 0.229 \\
\hline [10] & private & $\begin{array}{l}\text { GFMMNN } \\
(\text { Fuzzy+ANN) }\end{array}$ & ADL(General) & $\begin{array}{lr}\text { Current } & \text { error } \\
\text { (Missed / No } & \\
\text { Presentations) } & \end{array}$ & $\begin{array}{l}\text { Adapted: } 0.01 \text { for } 220 \text { online } \\
\text { presentations }\end{array}$ \\
\hline [5] & private & $\begin{array}{l}\text { Evolving } \\
\text { fuzzy classifi- } \\
\text { ers }\end{array}$ & ADL(General) & F-measure & $60 \%-70 \%$ \\
\hline$[12]$ & private & GT2FC(Fuzzy) & ADL(General) & Accuracy & $\begin{array}{l}\text { For } 70 \% \text { labelled data accur- } \\
\text { acy is } 81.65 \%\end{array}$ \\
\hline [23] & public & HMM & ADL(Healthcare) & Accuracy & $\begin{array}{l}90.75 \% \text { behaviour-level re- } \\
\text { cognition accuracy, } 98.45 \% \\
\text { observation-level recognition } \\
\text { accuracy }\end{array}$ \\
\hline [112] & private & HSMM & ADL(General) & F-measure & $65.5 \%$ \\
\hline$[45]$ & private & EPs & ADL(General) & $\begin{array}{l}\text { Time-slice accur- } \\
\text { acy }\end{array}$ & $85.84 \%$ \\
\hline [93] & public & $\begin{array}{l}\text { Ontological } \\
\text { approach }\end{array}$ & ADL(General) & Accuracy & $80.3 \%$ \\
\hline
\end{tabular}

In many cases, the resident of an SH for healthcare has special design requirements. For example, the limitation of short-term memory is more severe for elderly people and on some occasions, it is difficult for them to learn new interaction methods. Consequently, an SH's interface design process needs to reflect on additional human factors and use natural user interfaces (NUI) such as gesture recognition and speech recognition. Multi-modal NUI is a family of interfaces which are invisible to the users and allow them to communicate with the system in a way that they communicate with the world.

Orpwood et al. [82] presented an interface design methodology for people with dementia (Fig. 14). The study argued that the interface design is successful only if it is human-centred and led by users themselves. Considering demented people's memory condition, only caregivers were involved during the evaluation step. Several scenarios were studied to demonstrate the effectiveness of the methodology proposed. Many considera- tions have resulted from this study, including caregiver emulation, familiarity of the user with the new interface/device, prompts and reminders, and patient behaviour monitoring.

In another study, Koskela et al. [62] carried out a set of usability experiments to evaluate different interfaces for remote control of SHs. Three types of user interfaces were evaluated: a PC-based interface, a media-terminal interface (attached to the TV) and a mobile phone interface. The results stated that a PC-based interface was more appropriate for a central unit to control functions that can be planned and determined in advance (i.e., turn on the kitchen light every day at $8 \mathrm{am}$ ). The study showed that the mobile phone interfaces are more suitable for instant control (i.e., switch off the gas hob now). In six months of trial with two people, a young couple, the mobile phone was the primary and most frequently-used interface.

The Sweet-Home project [86] suggested a voice interface for elderly residents. The system was designed 
for control of windows blinds, lights, kitchen appliances, etc. The evaluation involved 18 participants consisting of eight old persons, seven informal caregivers (relatives) and three formal caregivers (professional caregivers). To assess the effectiveness of the system, a series of qualitative evaluation methods such as wizard of $\mathrm{OZ}$ and interviews were used. Results revealed that old residents prefer voice interfaces over other interaction methods such as typing or touch interfaces.

Rocker et al. [97] employed three qualitative research methods to collect the interface requirements based on: scenarios, focus group and open discussion. The study resulted in a set of prioritised design guidelines:

- The residents must always remain in charge of the system

- The system must be secure, safe and protect user's privacy

- The system must provide added value over existing ones

- The system should never unnecessarily replace direct interaction between people

- The home comfort should always be a priority and not be subversive to the system

In [73] a web-based interface for SHs, called Casalendar, was presented. Casalendar which visualizes $\mathrm{SH}$ data in the form of a calendar [time, event/task], was evaluated by 6 participants. Results illustrated that the participants were thoroughly in favour the calendar-like (temporal) interface. In [53] an interesting approach was presented. A "smart space" was created where objects, e.g., glass, table, digital photo frame, or MP3 player can be interactive. For instance an interactive table could perceive users' actions on a glass and triggers events like changing the music. The "smart space" was evaluated by some participants who provided positive feedbacks.

More interestingly, in [96] the authors reported on a prototype for elderly monitoring SH. In particular design aspects and core functionalities of the system architecture were described. The prototype is based service-oriented architecture (SOA) that addresses data distribution and scalability of SHs in healthcare. The prototype is equipped with web-interface that was evaluated by a certain number of people who rated it 7.8/10. The interface displays the sensor readings such as room temperature, fire sensors status and blood pressure. Also, it provided the users ability of configuring the SH based on the residents' preferences.

The study [77] introduced a set of requirements for SH user interfaces and relevant technologies. In particular it is stated that a user interface for domestic home applications should be simple, social, ethical and beautiful. The simplicity in design was studied in relation to people with dementia on how to make such people not forget which knob controls which stove. For the social requirement, the authors measured the enjoyment made by using services through a set of qualitative experiments. The ethical criterion in design was analysed in the tele-care context. Privacy was discussed from the perspective of agreement of the people through formal consent and responsibility of tele-care services. The "beauty" criterion was studied on two pieces of necklaces with monitoring sensors that could transmit signals to each other remotely. Unfortunately in this study no evaluation was done.

\section{Discussion}

This overview covers various technologies used to build SH systems. It summarizes sensing technologies, communication platforms, data processing techniques and user interfaces with illustrative research studies. The aim is to provide sufficient information to draw a broad picture of the state of the art technologies without intending to be exhaustive. In the following, we will discuss the challenges of design and implementation of SHs in general and in healthcare in particular. It addresses the challenges of data processing and particularly activity recognition that plays a significant role in the functionality of SHs.

\subsection{Security, Privacy and Reliability}

Many qualitative studies $[27,130]$ pointed out that privacy and the security are key concerns in SH technology. For instance, to investigate the importance of privacy, Demiris et al. [30] ran a qualitative study with 14 old persons living in a retirement home confirming that the major concern is the privacy, especially the use of video cameras at the home. On the other hand, wireless technologies raise more security issues compared to wired ones. Data collected from SHs is highly sensitive and therefore privacy measures have to be taken to meet the legal and ethical standards.

In [20], 6 major issues were identified: sensor compromise, eavesdropping, privacy of data, denial of service attacks, and malicious use of the sensor network and the limitations and advantages of the existed solutions for these issues were addressed. Kotz et al. [63] compared different existing privacy frameworks and proposed a set of privacy policies for mobile healthcare applications and SH systems. Some of these policies are:

- People should be informed on the matters related to collection and storage of data. 
- People should always be enabled to access their own data.

- Easy-to-use interfaces should be provided.

- The collection and storage of data should be restricted only for the purpose of monitoring.

- The quality of data in terms of completeness, authenticity and accuracy should be ensured.

- People's identity and personal data should be protected against unauthorised access.

Preserving reliability and safety is an important matter in SHs. The well-being of the occupant can be affected by the $\mathrm{SH}$ system itself if safety requirements are not considered during the design and development of the SH system. For instance, if a (vulnerable) occupant increases the heater temperature inadvertently to an unacceptable level, the consequence for him can be severe. To prevent critical problems, SH systems should be equipped with safety measures, especially in the case of vulnerable people [32]. Moreover, to develop stable $\mathrm{SH}$ systems, designers should use reliable components (software, hardware, communication protocols, etc.).

A challenging aspect of $\mathrm{SH}$ security is that conventional security methods and components i.e., authentication are often complicated for stakeholders in a dialogue of care such as patients, formal, informal and social caregivers. For instance, it is rather difficult for an elderly person with mild cognitive disorder to remember a complex passwords. On the other hand, most of $\mathrm{SH}$ data is quite sensitive and private, and requires an adequate security arrangement. Many research studies attempted to address these issues by employing novel security measures such as biometric and graphical passwords.

In [41], the benefits and drawbacks of biometric technologies for authentication in healthcare environment have been presented. The outcomes suggested that the main advantages of biometric technologies are the reliability of user authentication mechanisms, restriction in delegation of access rights as well as discouraging fraudulent access or impersonation of users. Moreover, the biometric authentication technologies facilitate the remote access to electronic health records for both patients and other stakeholders, reduces maintenance cost and provides a secure method for encryption of personal data. They also presented some limitations of biometric technologies such as the ability of a user can be influenced by age, skin colour, damage or lack of a biometric feature. Furthermore, biometric technologies could not be suitable for particular health settings. For example, fingerprint technology for laboratories and hygienic areas where user would be required to wear hygienic gloves.
Another feasible solution for authentication challenges in healthcare $\mathrm{SH}$ is to use video-based systems. Authors in [17] presented a prototype "mean of video event recognition protocol" for authentication. Evaluation of the prototype with an initial test of usability illustrated that that the authentication mechanism was well accepted by users and achieved a considerably low error rates.

In [54], a security architecture for healthcare systems was proposed. The key concern that authors attempted to address was the necessity of having an architecture that is secure while flexible. The approach security relies on a modern cryptographic scheme. The schema that is not entirely based on smart cards and allows the patient to authorize other stakeholders remotely (e.g., by phone) to access their healthcare data securely.

Authors in [15] proposed an entirely distributed peer to peer system that allows secure sharing of both medical and none-medical information for healthcare management. In the system, each node can access swiftly, easily and in a secure way to a substantial amount of data. Hence, the system advances the speed of clinical evaluation performed by the stakeholders in a dialogue of care. A group of physicians were asked to use the system with simulated data. For evaluating the participants' experience, they asked them questions regarding how the system affects and improve the existing systems. The responses to the questions were positive in all cases.

Graphical passwords are functioning by clicking on images rather than typing alphanumeric strings. These authentication methods have a great possibility of being used in healthcare systems since they are safer and simpler compared with conventional passwords. To make them even more secure, authors in [16] proposed a twofactor graphical password scheme and analysed the security and usability for an authentication mechanism based on it. The research illustrated that the time required to extract the user password in the method increases exponentially.

\subsection{Activity recognition}

While there has been an extensive work on activity recognition in the context of smart homes, not all computational models have been uniformly applied. Clearly the data-driven algorithms have been predominantly applied in various studies $[25,101,112]$ to model human activities. Moreover, even among the data-driven models, the probabilistic ones such as HMMs and alike have been the mostly used. Few authors have applied 
non-probabilistic models such as neural networks, decision trees, emerging patterns, etc.

Knowledge-driven techniques seem not to be very popular. Only very few authors have tried to apply logic and ontologies [93] to model activities. Some work mixed probabilistic models with knolwedge-based techniques as in $[51,52]$ where the evidential theory and ontologies are combined to handle uncertainty attached to activity recognition.

Reflecting on the approaches studied in the section 3 it can be assumed that selecting the best approach for constructing the activity model heavily depends on the problem that the authors focused on, while modelling the activities. The construction of the activity model is affected by numerous factors and can face many challenges. The followings are the challenges and open questions in the area of activity recognition.

- Recognizing interweaved and concurrent activities: It remains a challenging and active area of research. As an example, a study [45] adopted an EP-based approach to recognize these types of activities in addition to sequential ones. Some authors advised that $\mathrm{CRF}$ is a more flexible alternative to HMM when modelling these types of activities [61].

- Imbalanced data: The amount of data recorded for each activity in the dataset in the field of activity recognition is often imbalanced like the one described in [115]. There are many events belonging to the activity going to the bed rather than to the other activities. The study in [115] stated that HMM is more appropriate for imbalanced classes than CRF.

- Multi-resident activities: Most of the work on smart homes has been concerned with single-occupancy. However, in general a home is occupied by more than one person. In the simplest situation, a person lives alone but could have a pet and receive visitors such as family members, caregivers, friends, neighbors, etc. In presence of more than one occupant, many of the daily activities can be performed in parallel or together in a group. The modeling and recognition of such activities require a different approach from the ones used for single-occupancy activities. A few number of studies have recently focused on multi-occupancy [45,87], but the area is still to grow as it comes with is own scientific challenges and application potential.

- Online activity learning: In comparison to offline activity recognition, online activity recognition has not been much investigated by the researchers. In fact, most of the methods used by the researchers in the field are based on offline supervised learning. An interesting comparison study of online and offline inference using fuzzy rule based systems is presented in [12] showing that online learning performs equally well as offline learning. In [115] also a comparison between offline and online settings using HMM and CRF has been presented. However the online setting considered is not the usual setting used in online learning where only part of the data, ideally only the new sample(s) is used. The authors pointed out that the offline inference performed better for both models. Because of the relevance of online learning in this context of activity monitoring, it is important that more effort should be devoted to it.

- Applicability and adaptability of the activity model: Existing activity recognition systems were trained either on private datasets or publicly available datasets. The fundamental problem with these systems is that they require real-world data. Often such systems are trained on particular data stemming from a particular setting and therefore the activity recognition models are tightly tailored to the characteristics of the living space from which data has been collected, to a particular user's habits, and to the types of activities monitored at home. As a consequence, an activity recognition system trained in an environment would not be applicable to other environments. To overcome this problem, the authors in [101] suggested the use of an alternative source of activity data, such as web data, to train the activity model. However this solution does not reflect on the real-world situations.

- Scalability of the activity model: Scalability of the activity model is an important issue and can be discussed in terms of:

a- New activities: Considering scalability of activity models presented in section 3 , some of them present the advantage of having a separate model for each activity. As in [45] the authors mined a set of EPs for each activity. In the same context, the authors of the study [51,52] constructed an EON for each activity. Hence, adding a new activity in the environment only implies constructing the corresponding model for the latter (mining the set of EPs or constructing an EON respectively). Also, it would be easier to achieve high recognition rate with separate models than with a global model for all activities.

b- New residents: all the methods discussed here have focused on single-occupancy. Dealing with scalability in SHs should consider not only new activities, but also new occupants. Obviously, the scalability of the models with respect to the number of residents is the most important issue. Evaluating the activity model with more than 
one resident is an important aspect for real world situations.

\section{Conclusion}

While this survey paper does not claim to be exhaustive, it gives a fairly complete overview of the techniques and technologies involved in smart homes. The survey follows a logical structure inspired by the processing layers a smart home system consists of: sensing, processing, interacting. To the best of our knowledge there is no survey that outlines all these layers at the same time. While the survey tries to focus on the healthcare application of SHs, it does cover the methods and technologies in general terms regardless of the application.

Clearly SHs as a technology is not only a research topic, but it is being established as a product in the market. Because it is versatile, it can be applied for various purposes like tele-care, tele-health, comfort, energy saving, etc. These applications share basically the same design principles, but differ in terms of practical requirements. Each targeted application comes with its own and typical requirements that $\mathrm{SHs}$ have to consider. When developing SHs for a particular application, there are a lot of choices and therefore one has to take the specificity of that application into account. In other words, developing a SH system for healthcare is not the same as for comfort.

The literature overview presented in this paper sheds light on the main research areas including the hardware, the data analytics and artificial intelligence, and human-computer interfaces. It also discusses the potential and limitation of the approaches presented as well as various aspects like security, privacy, reliability and activity recognition. The survey highlighted also some of the open issues that still need further investigations.

\section{References}

1. G. D. Abowd, A. K. Dey, P. J. Brown, N. Davies, M. Smith, and P. Steggles. Towards a better understanding of context and context-awareness. In Handheld and ubiquitous computing, pages 304-307. Springer, 1999.

2. G. Acampora, D. J. Cook, P. Rashidi, and A. V. Vasilakos. A Survey on Ambient Intelligence in Healthcare. Proceedings of the IEEE, 101(12):2470-2494, 2013.

3. A. Ahmed, J. Ali, A. Raza, and G. Abbas. Wired Vs Wireless Deployment Support For Wireless Sensor Networks. In International technical conference of IEEE Region 10, TENCON, pages 1-3, 2006.

4. A. R. Al-Ali, M. A. Rousan, and M. Mohandes. GSMbased wireless home appliances monitoring \& control system. In Proceedings of International Conference on Information and Communication Technologies: From Theory to Applications., pages 237-238. IEEE, 2004.
5. J. Andreu and P. Angelov. An evolving machine learning method for human activity recognition systems. Journal of Ambient Intelligence and Humanized Computing, 4(2):195-206, 2013.

6. C. R. Baker, K. Armijo, S. Belka, M. Benhabib, V. Bhargava, N. Burkhart, A. Der Minassians, G. Dervisoglu, L. Gutnik, and M. B. Haick. Wireless Sensor Networks for Home Health Care. 21st International Conference on Advanced Information Networking and Applications Workshops AINAW '07., 2:832-837, 2007.

7. P. Baronti, P. Pillai, V. W. C. Chook, S. Chessa, A. Gotta, and Y. F. Hu. Wireless sensor networks: A survey on the state of the art and the 802.15. 4 and ZigBee standards. Computer communications, 30(7):16551695, 2007.

8. R. Begg and R. Hassan. Artificial neural networks in smart homes. In Designing Smart Homes, pages 146164. Springer, 2006.

9. C. Bisdikian. An overview of the Bluetooth wireless technology. Communications Magazine, IEEE, 39(12):86-94, 2001.

10. A. Bouchachia. Fuzzy classification in dynamic environments. Soft Computing, 15(5):1009-1022, 2011.

11. A. Bouchachia. Advances in Computational Intelligence, chapter Fuzzy Classifiers, To appear. World Scientific, 2015.

12. A. Bouchachia and C. Vanaret. GT2FC: an online growing interval type-2 self-learning fuzzy classifier. IEEE T. Fuzzy Systems, 22(4):999-1018, 2014.

13. S. Brownsell, S. Blackburn, and M. S. Hawley. An evaluation of second and third generation telecare services in older people's housing. Journal of Telemedicine and Telecare, 14(1):8-12, 2008.

14. K. E. Caine, W. A. Rogers, and A. D. Fisk. Privacy perceptions of an aware home with visual sensing devices. In Proceedings of the Human Factors and Ergonomics Society Annual Meeting, volume 49, pages 1856-1858. SAGE Publications, 2005.

15. A. Castiglione, C. D'Ambrosio, A. De Santis, and F. Palmieri. On secure data management in healthcare environment. In Innovative Mobile and Internet Services in Ubiquitous Computing (IMIS), 2013 Seventh International Conference on, pages 666-671. IEEE, 2013.

16. L. Catuogno and C. Galdi. Analysis of a two-factor graphical password scheme. International Journal of Information Security, 13(5):421-437, 2014.

17. L. Catuogno and C. Galdi. On user authentication by means of video events recognition. Journal of Ambient Intelligence and Humanized Computing, 5(6):909-918, 2014.

18. M. Caudill. Neural networks primer, part I. AI Expert, 2(12):46-52, 1987.

19. F. Cavallo, M. Aquilano, L. Odetti, M. Arvati, and M. C. Carrozza. A first step toward a pervasive and smart ZigBee sensor system for assistance and rehabilitation. In IEEE International Conference on Rehabilitation Robotics, ICORR., pages 632-637. IEEE, 2009.

20. H. Chan and A. Perrig. Security and privacy in sensor networks. Computer, 36(10):103-105, Oct. 2003.

21. M. Chan, E. Campo, Estève, and J. Fourniols. Smart homescurrent features and future perspectives. Maturitas, 64(2):90-7, Oct. 2009.

22. L. Chen and C. Nugent. Ontology-based activity recognition in intelligent pervasive environments. International Journal of Web Information Systems, 2009. 
23. S.-L. Chua, S. Marsland, and H. Guesgen. Behaviour recognition from sensory streams in smart environments. In A. Nicholson and X. Li, editors, AI 2009: Advances in Artificial Intelligence, volume 5866 of Lecture Notes in Computer Science, pages 666-675. Springer Berlin Heidelberg, 2009.

24. D. J. Cook and S. K. Das. How smart are our environments? An updated look at the state of the art. Pervasive and mobile computing, 3(2):53-73, 2007.

25. D. J. Cook, N. Narayanan, and P. Rashidi. Activity discovery and activity recognition: A new partnership. IEEE Transactions on Systems, Man and Cybernetics, 2013.

26. D. J. Cook, G. M. Youngblood, E. O. Heierman III, K. Gopalratnam, S. Rao, A. Litvin, and F. Khawaja. MavHome: An agent-based smart home. IEEE International Conference on Pervasive Computing and Communications (PerCom), 3:521-524, 2013.

27. K. L. C. Courtney. Privacy and senior willingness to adopt smart home information technology in residential care facilities. Methods of information in medicine, 47(1):76-81, 2008

28. S. Dagtas, G. Pekhteryev, and Z. Sahinoglu. MultiStage Real Time Health Monitoring via ZigBee in Smart Homes. In AINA Workshops (2), pages 782-786, 2007.

29. L. C. De Silva. Audiovisual sensing of human movements for home-care and security in a smart environment. International Journal on Smart Sensing and Intelligent Systems, 1(1):220-245, 2008.

30. G. Demiris, M. J. Rantz, M. Aud, K. Marek, H. Tyrer, M. Skubic, and A. Hussam. Older adults' attitudes towards and perceptions of'smart home'technologies: a pilot study. Informatics for Health and Social Care, 29(2):87-94, 2004.

31. S. Dengler, A. Awad, and F. Dressler. Sensor/actuator networks in smart homes for supporting elderly and handicapped people. In 21st International Conference on Advanced Information Networking and Applications Workshops, AINAW'07., volume 2, pages 863868. IEEE, 2007.

32. G. Dewsbury, B. Taylor, and M. Edge. Designing safe smart home systems for vulnerable people. Dependability in Healthcare Informatics, pages 65-70, 2001.

33. A. Diaz-Ramirez, F. N. Murrieta, J. A. Atempa, and F. A. Bonino. Non-intrusive Tracking of Patients with Dementia Using a Wireless Sensor Network. In IEEE International Conference on Distributed Computing in Sensor Systems (DCOSS), pages 460-465, 2013.

34. D. Ding, R. A. Cooper, P. F. Pasquina, and L. FiciPasquina. Sensor technology for smart homes. Maturitas, 69(2):131-136, 2011.

35. A. Dowrick and A. Southern. Dementia 2014: Opportunity for change. Alzheimer's Society publications, 2014 .

36. O. Er, N. Yumusak, and F. Temurtas. Chest diseases diagnosis using artificial neural networks. Expert Systems with Applications, 37(12):7648-7655, 2010.

37. E. Farella, A. Pieracci, and A. Acquaviva. Design and implementation of WiMoCA node for a body area wireless sensor network. In Systems Communications., pages 342-347, Aug. 2005.

38. G. Fenza, D. Furno, and V. Loia. Hybrid approach for context-aware service discovery in healthcare domain. Journal of Computer and System Sciences, 78(4):12321247, 2012.

39. P. Ferrari, A. Flammini, D. Marioli, and A. Taroni. IEEE802.11 sensor networking. IEEE Transactions on Instrumentation and Measurement, 55(2):615-619, 2006.

40. A. Fleury, M. Vacher, and N. Noury. SVM-based multimodal classification of activities of daily living in health smart homes: sensors, algorithms, and first experimental results. IEEE Transactions on Information Technology in Biomedicine, 14(2):274-283, Mar. 2010.

41. A. E. Flores Zuniga, K. T. Win, and W. Susilo. Biometrics for electronic health records. J. Med. Syst., 34(5):975-983, Oct. 2010.

42. V. Foo Siang Fook, T. Siew Choo, M. Jayachandran, J. Biswas, and Z. Daqing. An ontology-based context model in monitoring and handling agitation behavior for persons with dementia. In Fourth Annual IEEE International Conference on Pervasive Computing and Communications Workshops, PerCom Workshops, pages 5 pp. $-564,2006$.

43. T. Gruber. Ontology. Encyclopedia of database systems, pages 1963-1965, 2009.

44. T. Gu, H. K. Pung, and D. Q. Zhang. Toward an osgi-based infrastructure for context-aware applications. Pervasive Computing, IEEE, 3(4):66-74, 2004.

45. T. Gu, Z. Wu, X. Tao, H. K. Pung, and J. Lu. epsicar: An emerging patterns based approach to sequential, interleaved and concurrent activity recognition. In IEEE International Conference on Pervasive Computing and Communications, 2009. PerCom 2009., 2009.

46. H. Hagras, V. Callaghan, M. Colley, G. Clarke, A. Pounds-Cornish, and H. Duman. Creating an ambient-intelligence environment using embedded agents. Intelligent Systems, IEEE, 19(6):12-20, 2004.

47. H. Hagras, F. Doctor, V. Callaghan, and A. Lopez. An Incremental Adaptive Life Long Learning Approach for Type-2 Fuzzy Embedded Agents in Ambient Intelligent Environments. IEEE Transactions on Fuzzy Systems, 15(1):41-55, 2007.

48. M. E. Hazen. The Technology Behind HomePlug AV Powerline Communications. Computer, 41(6):90-92, 2008.

49. Z. He and L. Jin. Activity recognition from acceleration data based on discrete consine transform and SVM. In IEEE International Conference on Systems, Man and Cybernetics, SMC., pages 5041-5044, Oct. 2009.

50. S. Helal, W. Mann, H. El-Zabadani, J. King, Y. Kaddoura, and E. Jansen. The gator tech smart house: A programmable pervasive space. Computer, 38(3):50-60, 2005.

51. X. Hong and C. D. Nugent. Implementing evidential activity recognition in sensorised homes. Technology and Health Care, 2011.

52. X. Hong and C. D. Nugent. Segmenting sensor data for activity monitoring in smart environments. Personal and ubiquitous computing, 2013.

53. Y.-C. Huang, K.-Y. Wu, and Y.-T. Liu. Future home design: an emotional communication channel approach to smart space. Personal and ubiquitous computing, 17(6):1281-1293, 2013.

54. T. Hupperich, H. Löhr, A.-R. Sadeghi, and M. Winandy. Flexible patient-controlled security for electronic health records. In Proceedings of the 2nd ACM SIGHIT International Health Informatics Symposium, pages 727-732. ACM, 2012.

55. S. S. Intille, K. Larson, J. Beaudin, E. M. Tapia, P. Kaushik, J. Nawyn, and T. J. McLeish. The PlaceLab: A live-in laboratory for pervasive computing research (video). In Proceedings of PERVASIVE Video Program, 2005. 
56. Y. Isoda, S. Kurakake, and H. Nakano. Ubiquitous sensors based human behavior modeling and recognition using a spatio-temporal representation of user states. In 18th International Conference on Advanced Information Networking and Applications, AINA., volume 1, pages 512-517 Vol.1, 2004.

57. S. Jiang, Y. Cao, S. Iyengar, P. Kuryloski, R. Jafari, Y. Xue, R. Bajcsy, and S. Wicker. CareNet: an integrated wireless sensor networking environment for remote healthcare, 2008.

58. A. Juels. RFID security and privacy: A research survey. IEEE Journal on Selected Areas in Communications,, 24(2):381-394, 2006.

59. N. Kasabov. Evolving connectionist systems. Springer, 2007.

60. J. Khan, J. S. Wei, M. Ringner, L. H. Saal, M. Ladanyi, F. Westermann, F. Berthold, M. Schwab, C. R. Antonescu, and C. Peterson. Classification and diagnostic prediction of cancers using gene expression profiling and artificial neural networks. Nature medicine, 7(6):673679, 2001.

61. E. Kim, S. Helal, and D. J. Cook. Human activity recognition and pattern discovery. Pervasive Computing, IEEE, 2010.

62. T. Koskela and K. Väänänen-Vainio-Mattila. Evolution towards smart home environments: empirical evaluation of three user interfaces. Personal and Ubiquitous Computing, 8(3-4):234-240, 2004.

63. D. Kotz, S. Avancha, and A. Baxi. A Privacy Framework for Mobile Health and Home-care Systems. In Proceedings of the First ACM Workshop on Security and Privacy in Medical and Home-care Systems, pages 1-12. ACM, 2009.

64. P. Kulkarni and Y. Ozturk. mPHASiS: Mobile patient healthcare and sensor information system. Journal of Network and Computer Applications, 34(1):402-417, 2011.

65. X. H. B. Le, M. Di Mascolo, A. Gouin, and N. Noury. Health smart home for elders-a tool for automatic recognition of activities of daily living. In 30th Annual International Conference of the IEEE Engineering in Medicine and Biology Society EMBS., pages 3316-3319. IEEE, 2008.

66. H. J. Lee, S. H. Lee, K.-S. Ha, H. C. Jang, W.-Y. Chung, J. Y. Kim, Y.-S. Chang, and D. H. Yoo. Ubiquitous healthcare service using Zigbee and mobile phone for elderly patients. International Journal of Medical Informatics, 78(3):193-198, 2009.

67. J.-S. Lee, Y.-W. Su, and C.-C. Shen. A Comparative Study of Wireless Protocols: Bluetooth, UWB, ZigBee, and Wi-Fi. In 33rd Annual Conference of the IEEE on Industrial Electronics Society, IECON., pages 4651, Nov. 2007.

68. H. Li, Q. Zhang, and P. Duan. A novel one-pass neural network approach for activities recognition in intelligent environments. In 7th World Congress on Intelligent Control and Automation, WCICA, pages 50-54. IEEE, 2008.

69. P. J. Lisboa and A. F. G. Taktak. The use of artificial neural networks in decision support in cancer: A systematic review. Neural Networks, 19(4):408-415, 2006.

70. A. Lotfi, C. Langensiepen, S. Mahmoud, and M. Akhlaghinia. Smart homes for the elderly dementia sufferers: identification and prediction of abnormal behaviour. Journal of ambient intelligence and humanized computing, 3(3):205-218, 2012.
71. M. Luštrek and B. Kaluža. Fall detection and activity recognition with machine learning. Informatica, 33(2):197-204, 2009.

72. E. D. Manley and J. S. Deogun. Location Learning for Smart Homes. In 21st International Conference on Advanced Information Networking and Applications Workshops AINAW '0\%., volume 2, pages 787$792,2007$.

73. S. Mennicken, J. Hofer, A. Dey, and E. M. Huang. Casalendar: a temporal interface for automated homes. In CHI'14 Extended Abstracts on Human Factors in Computing Systems, pages 2161-2166. ACM, 2014.

74. R. Messing, C. Pal, and H. Kautz. Activity recognition using the velocity histories of tracked keypoints. In IEEE 12th International Conference on Computer Vision, pages 104-111, Sept. 2009.

75. A. Mihailidis, J. N. Boger, T. Craig, and J. Hoey. The COACH prompting system to assist older adults with dementia through handwashing: An efficacy study. BMC geriatrics, 8(1):28, 2008.

76. F. G. Miskelly. Assistive technology in elderly care. Age and ageing, 30(6):455-458, 2001.

77. A. F. Monk. Simple, social, ethical and beautiful: requirements for uis in the home. In Proceedings of the ninth conference on Australasian user interface-Volume 76, pages 3-9. Australian Computer Society, Inc., 2008.

78. M. C. Mozer. The neural network house: An environment that adapts to its inhabitants. Proceedings of the American Association for Artificial Intelligence, 1998.

79. N. Murata, S. Yoshizawa, and S.-I. Amari. Network information criterion-determining the number of hidden units for an artificial neural network model. IEEE Transactions on Neural Networks, 5(6):865-872, 1994.

80. N. Noury and T. Hadidi. Computer simulation of the activity of the elderly person living independently in a Health Smart Home. Computer Methods and Programs in Biomedicine, 108(3):1216-1228, 2012.

81. F. J. Ordonez, J. A. Iglesias, P. de Toledo, A. Ledezma, and A. Sanchis. Online activity recognition using evolving classifiers. Expert Systems with Applications, 40(4):1248-1255, 2013.

82. R. Orpwood, C. Gibbs, T. Adlam, R. Faulkner, and D. Meegahawatte. The design of smart homes for people with dementi a user-interface aspects. Universal Access in the Information Society, 4(2):156-164, 2005.

83. C. Orwat, A. Graefe, and T. Faulwasser. Towards pervasive computing in health careA literature review. BMC Medical Informatics and Decision Making, 8(1):26, 2008

84. M. Patel and W. Jianfeng. Applications, challenges, and prospective in emerging body area networking technologies. Wireless Communications, IEEE, 17(1):80-88, 2010.

85. S. N. Patel, M. S. Reynolds, and G. D. Abowd. Detecting human movement by differential air pressure sensing in HVAC system ductwork: An exploration in infrastructure mediated sensing. In Pervasive Computing, pages 1-18. Springer, 2008.

86. F. Portet, M. Vacher, C. Golanski, C. Roux, and B. Meillon. Design and evaluation of a smart home voice interface for the elderly: acceptability and objection aspects. Personal and Ubiquitous Computing, 17(1):127$144,2013$.

87. M. Prossegger and A. Bouchachia. Multi-resident Activity Recognition Using Incremental Decision Trees. In Proceedings of the 3rd International Conference on Adaptive and Intelligent Systems ICAIS' 2014, pages 182191, 2014. 
88. J. R. Quinlan. Induction of Decision Trees. Machine Learning, 1(1):81-106, 1986.

89. M. Rantz, M. Skubic, S. Miller, and J. Krampe. Using technology to enhance aging in place. In Smart Homes and Health Telematics, pages 169-176. Springer, 2008.

90. N. Ravi, N. Dandekar, P. Mysore, and M. L. Littman. Activity recognition from accelerometer data. In Association for the Advancement of Artificial Intelligence (AAAI), volume 5, pages 1541-1546, 2005.

91. B. Reeder, E. Meyer, A. Lazar, S. Chaudhuri, H. J. Thompson, and G. Demiris. Framing the evidence for health smart homes and home-based consumer health technologies as a public health intervention for independent aging: A systematic review. International Journal of Medical Informatics, 82(7):565-579, 2013.

92. D. Riboni and C. Bettini. Cosar: hybrid reasoning for context-aware activity recognition. Personal and Ubiquitous Computing, 15(3):271-289, 2011.

93. D. Riboni, L. Pareschi, L. Radaelli, and C. Bettini. Is ontology-based activity recognition really effective? Pervasive Computing and Communications Workshop, 2011.

94. D. E. Riedel, S. Venkatesh, and W. Liu. Spatial Activity Recognition in a Smart Home Environment using a Chemotactic Model. In Proceedings of the International Conference on Intelligent Sensors, Sensor Networks and Information Processing Conference, pages 301-306, 2005.

95. F. Rivera-Illingworth, V. Callaghan, and H. Hagras. A neural network agent based approach to activity detection in AmI environments. IEEE international workshop on intelligent environments, pages v2:92-v2:92, 2005.

96. J. Rivero-Espinosaa, A. Iglesias-Péreza, J. A. GutiérrezDueñasa, and X. Rafael-Paloub. Saapho: an aal architecture to provide accessible and usable active aging services for the elderly. ACM SIGACCESS Accessibility and Computing, (107):17-24, 2013.

97. C. Rocker, M. D. Janse, N. Portolan, and N. Streitz. User Requirements for Intelligent Home Environments: A Scenario-driven Approach and Empirical Crosscultural Study. In Proceedings of the 2005 Joint Conference on Smart Objects and Ambient Intelligence: Innovative Context-aware Services: Usages and Technologies, pages 111-116. ACM, 2005.

98. L. Rokach and O. Maimon. Decision Trees. In O. Maimon and L. Rokach, editors, Data Mining and Knowledge Discovery Handbook, chapter 9, pages 165-192. Springer US, 2005.

99. F. Sadri. Ambient intelligence. ACM Computing Surveys, 43(4):1-66, Oct. 2011.

100. C. H. Salvador, M. P. Carrasco, M. G. de Mingo, A. M. Carrero, J. M. Montes, M. A. Martin, L Sosa and Cavero, I. F. Lozano, and J. L. Monteagudo. Airmedcardio: a GSM and Internet services-based system for out-of-hospital follow-up of cardiac patients. IEEE Transactions on Information Technology in Biomedicine, 9(1):73-85, 2005.

101. A. M. J. Sarkar, Y.-K. Lee, and S. Lee. ARHMAM: an activity recognition system based on hidden Markov minded activity model. Proceedings of the 4th International Conference on Uniquitous Information Management and Communication, 2010.

102. P. Smith. Comparisons between low power wireless technologies. US Patent CS-213199-AN, 2011.

103. I. Sommerville. Software Engineering. Pearson Addison Wesley, 2004.
104. N. K. Suryadevara and S. C. Mukhopadhyay. Wireless Sensor Network Based Home Monitoring System for Wellness Determination of Elderly. Sensors Journal, IEEE $12(6): 1965-1972,2012$.

105. C. Sutton and A. McCallum. An introduction to conditional random fields for relational learning. Introduction to statistical relational learning, 2006.

106. E. Tapia, S. Intille, and K. Larson. Activity Recognition in the Home Using Simple and Ubiquitous Sensors. In A. Ferscha and F. Mattern, editors, Pervasive Computing, volume 3001, chapter 10, pages 158-175. Springer Berlin Heidelberg, 2004.

107. G. Tia, T. Massey, L. Selavo, D. Crawford, C. Borrong, K. Lorincz, V. Shnayder, L. Hauenstein, F. Dabiri, J. Jeng, A. Chanmugam, D. White, M. Sarrafzadeh, and M. Welsh. The Advanced Health and Disaster Aid Network: A Light-Weight Wireless Medical System for Triage. IEEE Transactions on Biomedical Circuits and Systems, 1(3):203-216, 2007.

108. S. Tompros, N. Mouratidis, H. Hrasnica, and M. Caragiozidis. A novel power line network architecture for managing the energy resources of the residential environment. In IEEE International Symposium on Power Line Communications and Its Applications. ISPLC, pages 211-216, 2009.

109. W. Trumler, F. Bagci, J. Petzold, and T. Ungerer. Smart doorplate. Personal and Ubiquitous Computing, 7(3-4):221-226, 2003.

110. J. Van Hoof, H. Kort, P. Rutten, and M. Duijnstee. Ageing-in-place with the use of ambient intelligence technology: Perspectives of older users. International journal of medical informatics, 80(5):310-331, 2011.

111. T. L. van Kasteren. Activity recognition for health monitoring elderly using temporal probabilistic models. $\mathrm{PhD}$ thesis, University Van Amstrdam, 2011.

112. T. L. van Kasteren, G. Englebienne, and B. J. Krose. Activity recognition using semi-markov models on real world smart home datasets. Journal of ambient intelligence and smart environments, 2010.

113. T. L. van Kasteren, Gwenn, and B. J. Krose. Human activity recognition from wireless sensor network data: Benchmark and software. In Activity Recognition in Pervasive Intelligent Environments, pages 165-186. Springer, 2011.

114. T. L. van Kasteren and B. Krose. Bayesian activity recognition in residence for elders. IET Conference Proceedings, pages 209-212(3), Jan. 2007.

115. T. L. van Kasteren, A. Noulas, G. Englebienne, and B. Kröse. Accurate activity recognition in a home setting. Proceedings of the 10th international conference on Ubiquitous computing, pages 1-9, 2008.

116. F. Viani, F. Robol, A. Polo, P. Rocca, G. Oliveri, and A. Massa. Wireless architectures for heterogeneous sensing in smart home applications: Concepts and real implementation. Proceedings of the IEEE, 101(11):23812396, 2013.

117. J. L. Wiles and R. Jayasinha. Care for place: The contributions older people make to their communities. Journal of aging studies, 27(2):93-101, 2013.

118. D. Wilson and C. Atkeson. Automatic health monitoring using anonymous, binary sensors. In CHI Workshop on Keeping Elders Connected, pages 1719-1720. Citeseer, 2004.

119. D. Wilson and C. Atkeson. Simultaneous tracking and activity recognition (STAR) using many anonymous, binary sensors. Pervasive computing, 3468:62-79, 2005. 
120. A. Wimo, B. Winblad, and L. Jonsson. An estimate of the total worldwide societal costs of dementia in 2005. Alzheimer's \&f Dementia, 3(2):81-91, 2007.

121. K. Wongpatikaseree, M. Ikeda, M. Buranarach, T. Supnithi, A. O. Lim, and Y. Tan. Activity recognition using context-aware infrastructure ontology in smart home domain. In Knowledge, Information and Creativity Support Systems (KICSS), 2012 Seventh International Conference on, pages 50-57. IEEE, 2012.

122. A. Wood, J. A. Stankovic, G. Virone, L. Selavo, Z. He, Q. Cao, T. Doan, Y. Wu, L. Fang, and R. Stoleru. Context-aware wireless sensor networks for assisted living and residential monitoring. Network, IEEE, 22(4):26-33, 2008.

123. T. Yamazaki. Beyond the Smart Home. In International Conference on Hybrid Information Technology ICHIT., volume 2, pages 350-355, 2006.

124. T. Yamazaki. The ubiquitous home. International Journal of Smart Home, 1(1):17-22, 2007.

125. J. Ye, S. Dobson, and S. McKeever. Situation identification techniques in pervasive computing: A review. Pervasive and mobile computing, 8(1):36-66, 2012.

126. M. S. Yousuf, S. Z. Rizvi, and M. El-Shafei. Power line communications: An overview-Part II. In 3rd International Conference on Information and Communication Technologies: From Theory to Applications. ICTTA, pages 1-6. IEEE, 2008.

127. L. Yu-Ju, H. A. Latchman, L. Minkyu, and S. Katar. A power line communication network infrastructure for the smart home. Wireless Communications, IEEE, 9(6):104-111, 2002.

128. L. A. Zadeh. Fuzzy sets. Information and control, 8(3):338-353, 1965.

129. J. Zhaohui, L. Lili, H. Xiaowei, and T. Chunjie. Design of wearable home health care system with emotion recognition function. In International Conference on Electrical and Control Engineering (ICECE), pages 2995-2998, 2011.

130. S. A. Zwijsen, A. R. Niemeijer, and C. M. P. M. Hertogh. Ethics of using assistive technology in the care for community-dwelling elderly people: An overview of the literature. Aging and mental health, 15(4):419-427, 2011. 